OPEN ACCESS

Edited by:

Jing Ma,

Shanghai JiaoTong University, China

Reviewed by:

Kathy L. Gatford,

University of Adelaide, Australia

Francesca Battista,

University of Padua, Italy

*Correspondence:

Claudia Torres-Farfan

claudia.torres@uach.cl

Specialty section:

This article was submitted to

Obesity,

a section of the journal

Frontiers in Endocrinology

Received: 09 March 2021

Accepted: 19 July 2021

Published: 16 August 2021

Citation:

Halabi D, Richter HG, Mendez N,

Kähne T, Spichiger C, Salazar E,

Torres F, Vergara K, Seron-Ferre $M$ and Torres-Farfan C (2021) Maternal

Chronodisruption Throughout

Pregnancy Impairs Glucose

Homeostasis and Adipose Tissue Physiology in the Male Rat Offspring.

Front. Endocrinol. 12:678468.

doi: 10.3389/fendo.2021.678468

\section{Maternal Chronodisruption} Throughout Pregnancy Impairs Glucose Homeostasis and Adipose Tissue Physiology in the Male Rat Offspring

\author{
Diego Halabi ${ }^{1,2}$, Hans G. Richter ${ }^{1}$, Natalia Mendez ${ }^{1}$, Thilo Kähne ${ }^{3}$, Carlos Spichiger ${ }^{4}$, \\ Esteban Salazar ${ }^{1}$, Fabiola Torres ${ }^{1}$, Karina Vergara ${ }^{1}$, Maria Seron-Ferre ${ }^{5}$ \\ and Claudia Torres-Farfan ${ }^{1 *}$ \\ ${ }^{1}$ Laboratory of Developmental Chronobiology, Institute of Anatomy, Histology and Pathology, Faculty of Medicine, \\ Universidad Austral de Chile, Valdivia, Chile, ${ }^{2}$ Institute of Dentistry, Faculty of Medicine, Universidad Austral de Chile, \\ Valdivia, Chile, ${ }^{3}$ Mass Spectrometry for Massive Proteomics, Institute of Experimental Internal Medicine, Medical Faculty, \\ Otto von Guericke University, Magdeburg, Germany, ${ }^{4}$ Institute of Biochemistry and Microbiology, Faculty of Sciences, \\ Universidad Austral de Chile, Valdivia, Chile, ${ }^{5}$ Programa de Fisiopatología, Instituto de Ciencias Biomédicas (ICBM), Facultad \\ de Medicina, Universidad de Chile, Santiago, Chile
}

Compelling evidence in rats support the idea that gestational chronodisruption induces major changes in maternal circadian rhythms and fetal development and that these changes impact adult life at many physiological levels. Using a model of chronic photoperiod shifting throughout gestation (CPS), in which pregnant female rats (Sprague-Dawley strain; $n=16$ per group) were exposed to lighting schedule manipulation every 3-4 days reversing the photoperiod completely or light/dark photoperiod (12/12; LD), we explored in the adult rat male offspring body weight gain, glucose homeostasis, adipose tissue content, adipose tissue response to norepinephrine $(\mathrm{NE})$, and adipose tissue proteomic in the basal condition with standard diet (SD) and in response to high-fat diet (HFD). In adult CPS male (100-200 days old; $n=8$ per group), we found increasing body weight, under SD and adiposity. Also, we found an increased response to intraperitoneal glucose (IGTT). After 12 weeks of HFD, white adipose tissue depots in CPS offspring were increased further, and higher IGTT and lower intraperitoneal insulin tolerance response were found, despite the lack of changes in food intake. In in vitro experiments, we observed that adipose tissue (WAT and BAT) glycerol response to NE from CPS offspring was decreased, and it was completely abolished by HFD. At the proteomic level, in CPS adipose tissue, 275 proteins displayed differential expression, compared with LD animals fed with a standard diet. Interestingly, CPS offspring and LD fed with HFD showed 20 proteins in common (2 upregulated and 18 downregulated). Based on these common proteins, the IPA analysis found that two functional pathways were significantly altered by CPS: network 1 (AKT/ERK) and network 2 (TNF/IL4; data are available via ProteomeXchange with identifier PXD026315). The present data show that gestational chronodisruption induced deleterious effects in adipose tissue recruitment and 
function, supporting the idea that adipose tissue function was programmed in utero by gestational chronodisruption, inducing deficient metabolic responses that persist into adulthood.

Keywords: DoHAD, adipose tissue, diabetes, obesity, chronobiology

\section{INTRODUCTION}

At present, according to the European Foundation for the improvement of living and working conditions and the US Department of Labor, approximately $20 \%$ of the worldwide workforce is employed under shift work schedule $(1,2)$, increasing a risk of early onset of a series of noncommunicable diseases (NCD), like metabolic syndrome, obesity, and cardiovascular diseases (1, 3-5). In this regard, compelling evidence in human and animal models support the idea that chronodisruption (i.e., disturbance of internal temporal order, essentially circadian, of endocrinology, physiology, metabolism, and behavior) might be a link between NCD and shift work schedule. Indeed, similar phenomena arose during pregnancy, namely, gestational chronodisruption (6-9). In human, exposure to shift work schedule during pregnancy has been associated with an increased risk of miscarriage, preterm delivery, and low birth weight, in addition to higher incidence of sleep and metabolic and cardiovascular disturbances in the offspring (10-12). Unfortunately, interpreting human studies during pregnancy is quite difficult, due to confusing scenarios imposed by the effects of food availability, electronic screen exposure, emotional support, and family life. Actually, new epidemiological studies add more evidence about the potential deleterious effects of gestational chronodisruption in human, although conducting studies with more controlled conditions is imperative $(5,13,14)$.

Therefore, to dissect the potential mechanism involved in the long-term effect of chronodisruption, we use a model in which pregnant female rats were exposed to lighting schedule manipulation every 3-4 days reversing completely their photoperiod (chronic photoperiod shift, CPS), simulating night shift work schedules in humans $(6,9)$. In vivo and in vitro experiments in rat, non-human primates, and sheep demonstrated that gestational chronodisruption affects fetal organ function like adrenal, heart, hippocampus, liver, and fetal hormonal rhythms like prolactin, corticosterone, and cortisol. Altogether, the current evidence supports the idea that gestational chronodisruption is indeed an unhealthy signal for fetal development. Moreover, adult offspring of these studies show changes in organs such as liver, kidneys, and pancreas and an increase in the amount of adipose tissue (6-8, 15-20). Interestingly, in precocious species like sheep, new information support the notion that the timing at which the effect of chronodisruption appears could be related to age. In a recent study, Gatford et al. (21) found a lack of or a weak effect of maternal chronodisruption in young sheep, in contrast to that reported previously in young rats (9).
Adipose tissue has been proposed as an important target of developmental programming since obesity is the main risk factor for numerous pathologies, such as type 2 diabetes mellitus, insulin resistance (22), hypertension (23), cardiometabolic disease (24), and some types of cancer (25). Obesity is observed clinically by an excessive accumulation of white adipose tissue, related to a state of chronic and mild inflammation, which is directly related to the complications generated by obesity (26). It must be kept in mind that in mammals, there are two types of adipose tissue that are structurally and functionally different: white adipose and brown adipose tissue. White adipose tissue (WAT) is responsible for energy storage in the form of triglycerides, and it plays an endocrine function through the secretion of inflammatory cytokines. Meanwhile, brown adipose tissue (BAT) is responsible for energy dissipation as heat, which is produced from triglycerides stored in brown adipocytes, a physiological process called thermogenesis $(27,28)$.

Using the rat model of CPS throughout gestation, we investigated the long-term detrimental effects of gestational chronodisruption on glucose homeostasis and adipose tissue physiology and metabolism in the male adult offspring, based on our previous studies in rat male offspring in which we documented the long-term effect of the cardiovascular, endocrine, and metabolic impact of gestational chronodisruption in the offspring (6). In this model, as in other models of developmental origin of health and diseases (29), important sex differences have been shown in the offspring (9). Furthermore, considering risk factors prevailing in a modern society subjected to night shift work schedules, the impact of a second cardiometabolic challenge [high-fat diet (HFD) for 12 weeks] was evaluated in the male adult offspring that had been gestated under CPS relative to LD (control) conditions. An integrative array of methodologies was applied to generate the following outcomes: food intake and cumulative weight gain, glucose and insulin tolerance, fasting glucose and insulin levels, serum leptin and adiponectin levels, WAT and BAT tissue and cellular characterization, in vitro WAT and BAT glycerol response to norepinephrine, global genomic DNA methylation, and quantitative proteomics analyses.

\section{MATERIALS AND METHODS}

\section{Animals}

The protocols were approved by the Bioethics Commission from the Universidad Austral de Chile (CBA: 352/2019). Animal handling was performed following the guidelines for the care 
and use of laboratory animals of the Institute for Laboratory Animals Research of the National Research Council.

We raised and maintained 32 female rats of Sprague-Dawley strain obtained from Charles River (CRL International Inc., Kingston NY). These rats were mated, and the pregnancy was determined by the presence of positive sperm, obtained by vaginal smears, calling that day "day 0 of gestation" (E0). All pregnant rats had water and standard food (Prolab ${ }^{\circledR}$ RMH 3000, Lab diet, USA) ad libitum under controlled temperature $\left(20^{\circ} \mathrm{C}-\right.$ $22^{\circ} \mathrm{C}$ ) in standard cages inside a cabinet with filters and ventilation. From day 1 of gestation, pregnant rats were randomly separated into two groups of light/dark photoperiod:

A) Control light/dark (LD $n=16$ pregnant female): From day 1 of gestation, a group of dams continued with the light/dark 12:12 photoperiod, with artificial white light $\sim 400$ lux at the head level, which is turned on at $0700 \mathrm{~h}$ and turned off at $1900 \mathrm{~h}$.

B) Chronic phase shift of photoperiod (CPS $n=16$ pregnant females): using a similar protocol reported by us (6). Briefly, pregnant females were exposed to lighting schedule manipulation every 3-4 days reversing completely the photoperiod. The photoperiod reversal occurred at the night of day 0 of gestation, so that lights, rather than going off at $1900 \mathrm{~h}$, remained on until $0700 \mathrm{~h}$ of day 2. At 18 days of gestation, the mothers returned to a normal 24-h photoperiod (12:12, lights on at $0700 \mathrm{~h}$ ) and continued in this photoperiod thereafter. Exposure of pregnant females to CPS had no impact on food consumption and maternal weight (6).

Exposure of pregnant females to CPS had no impact on daily food consumption (monitored every week) through pregnancy
(LD: $21.2 \pm 0.6 \mathrm{~g} /$ day, $n=16$ vs. CPS: $22.3 \pm 0.6, n=16$ ), maternal weight at the end of gestation (LD: $427.6 \pm 6.8 \mathrm{~g}, n=16$ vs. CPS: $430.3 \pm 10.6, n=16)$, or maternal weight gain at the end of gestation (LD: $134.4 \pm 2.8 \mathrm{~g}, n=16$ vs. CPS: $135.0 \pm 7.5 \mathrm{~g}, n=16$ ) as reported previously (6).

At birth, mothers and their pups were maintained in control photoperiod 12:12, and the male offspring were studied. All the animals were housed individually after weaning and weighted weekly, and food consumption was measured by weighing the food placed in the cage and the amount left 3 days later, when bed and fresh food were replaced. For the current study, we generated two cohorts of animals separated and used them in the protocols described below (Figure 1). At 100 days of age, two brothers from the same pre-natal condition were separated and one brother was exposed to a HFD (45\% excess calories) for 12 weeks and paired with a brother fed with standard food (Prolab ${ }^{\circledR}$ RMH 3000, Lab diet, USA). Intraperitoneal glucose tolerance test (iGTT, Cohort1) and intraperitoneal insulin tolerance test (iITT, Cohort 2) were performed at 100 days (basal) and 12 weeks after exposure to either a HFD or standard diet. In addition, only in those males exposed to HFD was an additional iGTT and iITT test performed after 6 weeks under HFD. One week after the last test, animals were euthanized and adipose tissue was collected.

\section{Intraperitoneal Glucose Tolerance Test}

Male rats ( $n=8$ per group-Cohort 1$)$ at $0800 \mathrm{~h}$ (after 12-h fasting) were anesthetized (Isoflurane $2.5 \%-3.5 \%$ ) and injected with intraperitoneal glucose (1 g/kg; Glucose, Sanderson laboratories; Chile). A blood drop was collected from the tail
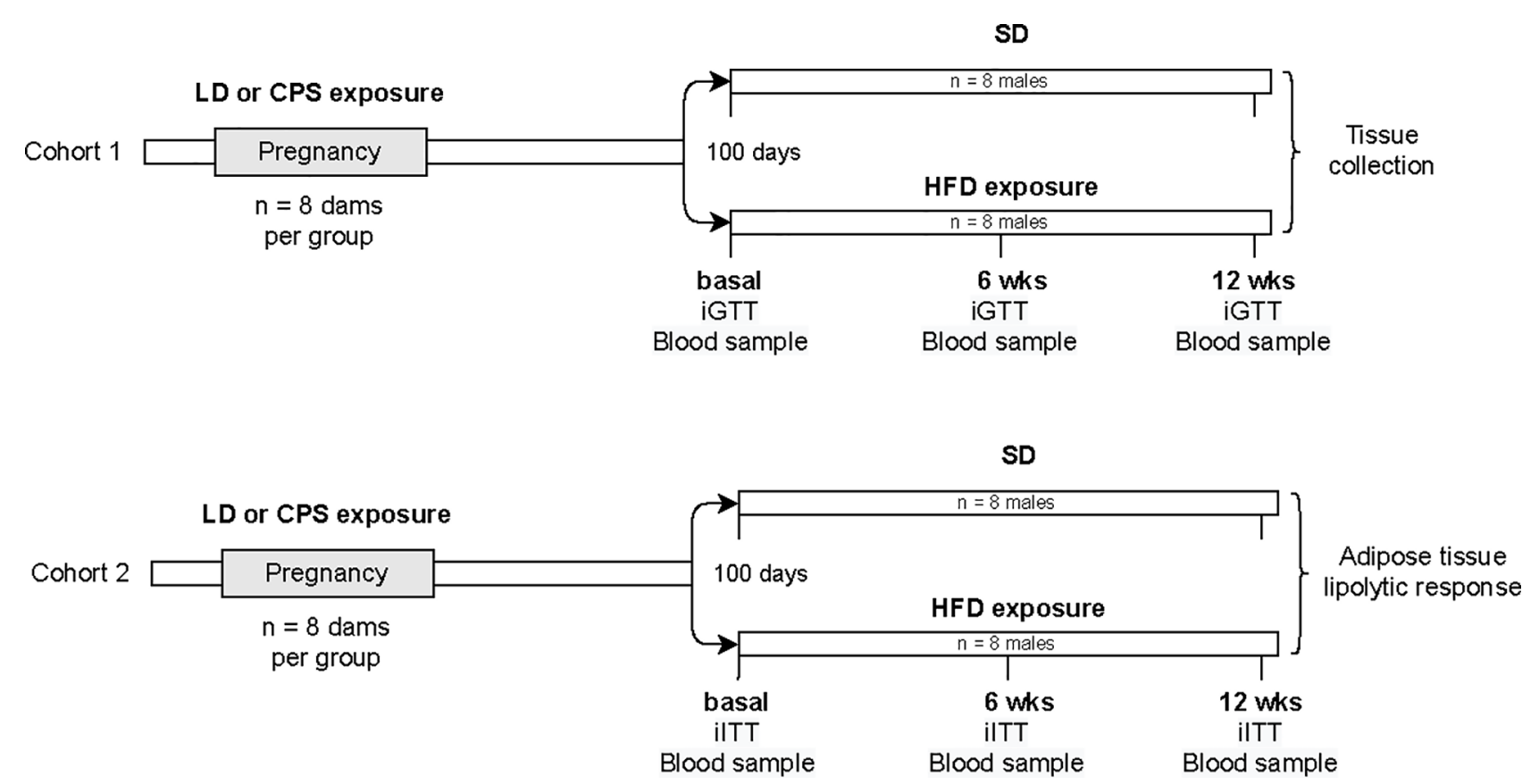

FIGURE 1 | Flow diagram of experimental protocol. Pregnant rats were exposed to either control photoperiod (LD) or chronic photoperiod shift (CPS) from mating until 18 days of gestation. At 100 days old, male offspring were exposed to either high-fat diet (HFD; 45\% excess calories) or standard diet (SD) for 12 weeks (about 200 days old). iGTT, intraperitoneal glucose tolerance test; ilT, intraperitoneal insulin tolerance test; $n=$ number of animals. 
to measure glucose levels (Accu-Chek; Roche Diagnostics) at -15 and 0 min before glucose administration and 30, 60, 90, 120, and $180 \mathrm{~min}$ after glucose injection. A blood sample was collected at $-15 \mathrm{~min}$ to measure fasting glucose, insulin, leptin and adiponectin.

\section{Intraperitoneal Insulin Tolerance Test}

Male rats ( $n=8$ per group-Cohort 2) at $1500 \mathrm{~h}$ were anesthetized and injected with insulin (1U/kg; Humalog ${ }^{\circledR}$ \#CAT: VL7510, Eli Lilly and Company, Indianapolis, USA) after 6-h fasting. A blood drop was collected from the tail to measure glucose levels (AccuChek; Roche Diagnostics) at 0, 15, 25, 35, 45, and 60 min after glucose injection. A blood sample was collected at $0 \mathrm{~min}$ to measure fasting glucose, insulin, leptin, and adiponectin.

\section{Adipose Tissue Depots}

At 200 days of age (after 12 weeks of HDF, $n=8$ per groupCohort 1), eight male rats per group were deeply anesthetized (Isoflurane 3\%, Baxter Laboratories, Melbourne, Australia), a midline incision was done to expose the vena cava, and an overdose of sodium thiopental (150 mg/kg; Vetpharma, Buenos Aires, Argentina) was administered. Immediately after death was confirmed, whole white adipose depots (inguinal, epigonadal, and perirenal) and interscapular brown adipose depot were dissected and weighted. Pieces were stored at $-80^{\circ} \mathrm{C}$ for proteomic analysis or fixed in $4 \%$ formalin for histological analysis. The remaining adipose tissue were stored in our tissue bank at $-80^{\circ} \mathrm{C}$.

\section{Adipose Tissue Lipolytic Response to Norepinephrine In Vitro}

( $n=8$ per group-Cohort 2 ). Epigonadal white adipose tissue (eWAT) and interscapular brown adipose tissue (iBAT) were dissected from individual rats, cut in small explants (about $50 \mathrm{mg}$ for eWAT and $25 \mathrm{mg}$ for iBAT) and suspended in culture medium (D-MEM F12, Sigma-Aldrich, St. Louis, MO, USA). Explants (10 explants for each animal) were pre-incubated in culture medium for $6 \mathrm{~h}$ at $37^{\circ} \mathrm{C}$ and aerated with $95 \%$ air $\mathrm{CO}_{2}$ and $5 \% \mathrm{O}_{2}$. Next, explants were incubated in duplicate for $6 \mathrm{~h}$ in $2 \mathrm{ml}$ medium alone (basal) or containing $0.01,0.1,1$, and $10 \mu \mathrm{M}$ of norepinephrine [A7257 (-)-Norepinephrine, Sigma-Aldrich, St. Louis, MO, USA]. At the end of incubation, the supernatant was collected, total protein of explants was assayed by Bradford method, and lipolysis was determined measuring the glycerol present in the supernatant fraction with a commercial kit (FG0100 Free Glycerol Determination Kit, Sigma-Aldrich, St. Louis, MO, USA). Production of glycerol was calculated as microgram per milligram of total protein in the explant.

\section{Hormone Assays}

Blood samples were collected from the tail of male rats $(n=8$ per group-Cohort 1 ) after 12 -h fasting ( $n=8$ per group) at basal, 6 weeks, and 12 weeks after HFD exposure. The serum of each sample was obtained by centrifugation $\left(4,000 \times g, 10 \mathrm{~min}, 22^{\circ} \mathrm{C}\right)$, and insulin, leptin, and adiponectin concentrations were measured by a commercial immunoassay kit according to the manufacturer's instructions (MILLIPLEX ${ }^{\circledR}$ MAP Kit; Merck,
KGaA, Darmstadt, Germany). The inter- and intra-assay coefficients were less than $10 \%$.

\section{Global Methylation}

Adipose tissue (prWAT and iBAT, $n=5 \mathrm{CPS}+\mathrm{SD}$ and LD + SD; 90 days old), stored in our tissue bank from previous studies (7, 30 ), were used to evaluate global genomic DNA methylation using MethylFlash (methylated DNA quantification colorimetric kit; Epigentek Group Inc., Farmingdale, NY, USA), following the manufacturer's instructions. Basically, our aim was to compare the effect of gestational chronodisruption in global methylation in adipose tissue since epigenetic changes have been reported previously by us in adrenal and kidney, organs in which such changes have been accompanied with changes in organ function already in fetal life.

\section{Proteomic Analyses}

Protein identification was performed by high-resolution mass spectrometry on a hybrid dual-pressure linear ion trap/orbitrap mass spectrometer (LTQ Orbitrap Velos Pro, Thermo Scientific, San Jose, CA, USA) equipped with an EASY-nLC Ultra HPLC (Thermo Scientific). Input was $50 \mathrm{mg}$ of white adipose tissue for each sample ( $n=8$ per group). For analysis, peptide samples were adjusted to $10 \mu \mathrm{l} 1 \% \mathrm{ACN} / 0.1 \%$ TFA and fractionated on a 75- $\mu \mathrm{m}$ (ID), 25-cm PepMap C18-column, packed with $2 \mu \mathrm{m}$ resin (Dionex/Thermo Scientific). The separation was achieved through applying a gradient from $2 \%$ to $35 \%$ ACN in $0.1 \%$ formic acid over $150 \mathrm{~min}$ at a flow rate of $300 \mathrm{nl} / \mathrm{min}$. An Orbitrap full MS scan was followed by up to 20 LTQ MS/MS runs using collision-induced dissociation (CID) fragmentation of the most abundantly detected peptide ions. Essential MS settings were as follows: full MS (FTMS; resolution 60,000; $\mathrm{m} / \mathrm{z}$ range 400-2000); MS/MS (Linear Trap; minimum signal threshold 500 ; isolation width $2 \mathrm{Da}$; dynamic exclusion time setting $30 \mathrm{~s}$; singly charged ions were excluded from selection). The normalized collision energy was set to $35 \%$, and activation time was set to $10 \mathrm{~ms}$. The mass spectrometry proteomics data have been deposited to the ProteomeXchange Consortium via the PRIDE (31) partner repository with the dataset identifier PXD026315.

\section{Statistical Analysis}

Data are expressed as mean \pm standard error of the mean (SEM). LD and CPS differences in weight gain, food consumption, glycerol release, hormone levels, iGTT, iITT, and global methylation were analyzed by two-way repeated measures ANOVA and Bonferroni post-hoc test when group factor or interaction factor was significant (32). Additionally, we calculated the area under the curve (AUC) for iGTT and area above the curve (AAC) for iITT. Statistical analyses were performed using GraphPad Prism (version 9; GraphPad Software Inc., San Diego, CA). Results were considered significant when $p<0.05$. For adipose tissue proteomics analysis, raw data processing and protein identification were performed using PEAKS Studio V.8.0 (Bioinformatics Solutions, Waterloo, Canada). False discovery rate was set to $<1 \%$. Labelfree quantification was performed using Progenesis QI for 
proteomics (Nonlinear Dynamics/Waters). Proteins with abundance ratios of $>1.5$ or $<1 / 1.5$ at $p<0.05$ were considered as significantly regulated. We applied an integrative and unbiased analysis approach for functional analysis of the proteins with significant changes in expression against the control group (LD offspring). Ingenuity Pathways Analysis (IPA) computes a score for each protein network according to the fit of that network to the user-defined set of "focus protein." The score is derived from a $p$-value and indicates the likelihood of the focus proteins in a network being found together due to random chance. A score of 2 indicates that there is a 1 in 100 chance that the focus proteins are together in a network due to random chance. Therefore, scores of 2 or higher have at least a 99\% confidence of not being generated by random chance alone [for details, see (17)]. First, we identified the modified proteins for the LD + HFD, CPS and CPS + HFD conditions compared to LD (fold change $>1.5 ; p<0.05$ ). After this exploratory analysis, we decided to focus on the common proteins for the CPS and LD + HFD groups, based on our observed results. Under this criterion, we selected the 20 proteins shown in Figure 8 and were grouped to build a new dataset and analyzed by IPA to generate the interactomes.

\section{RESULTS}

\section{Effects of Gestational CPS on the Offspring's Metabolic Status}

Between weaning (21 days old) and 100 days of age, CPS and LD animals had similar food consumption expressed as kcal/day (LD: $137 \pm 12 \mathrm{kcal} /$ day, $n=16$ versus CPS: $143 \pm 14, n=16$; $p=0.197$ unpaired $t$-test). However, CPS offspring was slightly heavier (LD: $602 \pm 51 \mathrm{~g}, n=16$ versus CPS: $615 \pm 50 \mathrm{~g}, n=16$; $p=$ 0.5 , unpaired $t$-test). As shown in Figure 2A, CPS animals fed a standard diet (CPS + SD) between 100 and 200 days became increasingly heavier than LD + ST animals. After the 4 weeks under HFD, LD + HFD and CPS + HFD animals showed a significant increase in body weight versus $\mathrm{LD}+\mathrm{SD}$ (time $\times$ group factor $p<0.001$; Figure 2A). Thus, CPS animals with SD or HFD featured a similar weight gain profile, reaching an increase of about $50 \%$ versus $\mathrm{LD}+\mathrm{SD}$ (Figure 2A). Food intake remains steady along the 12 weeks in animals receiving either standard or HFD (Figure 2B). Therefore, CPS animals fed with SD had a similar weight gain to those LD or CPS fed with HFD.

In line with a previous report (6), we found slight differences in intraperitoneal glucose tolerance test (iGTT) between LD and CPS adult offspring at 100 days of age, before the beginning of HFD (Figure 3A). After $60 \mathrm{~min}$ post glucose injection, glucose levels rapidly decreased to $173 \pm 2 \mathrm{mg} / \mathrm{dl}$ in the LD group, remaining elevated in the CPS group $(210 \pm 16 \mathrm{mg} / \mathrm{dl} ; p=$ 0.024; two-way ANOVA and Bonferroni test), but no interaction time $\times$ column was found $(p=0.172)$. In addition, analyzed as AUC, glucose response displayed a similar value to the CPS group without reaching statistical significance $(p=0.857$, unpaired $t$-test; Figure 3A). However, no difference was found at 200 days (Figure 4A). Important differences were found in iGTT after 6 weeks with HFD. At this time window, the adult offspring gestated under LD conditions maintained the glucose response similar to that observed at week 0 . In contrast, a marked increase in glucose response was observed in the CPS group relative to control LD (time $\times$ group factor: $p=0.012$, two-way ANOVA and Bonferroni; Figure 3C). Analyzed as AUC, glucose response remained the same as week 0 (basal) for the LD group, while basal plasma glucose levels tended to increase in the CPS group relative to control LD group ( $p=0.111$; unpaired $t$-test). As anticipated, a significant increase in glucose response was found in LD animals after 12 weeks (Figure 3C) regarding 0 and 6 weeks of HFD. At this late time window, the adult CPS offspring presented significantly higher levels of glucose in basal conditions, raising up to $340 \mathrm{mg} / \mathrm{dl}$ levels after $30 \mathrm{~min}$ post intraperitoneal glucose injection (Figure 3E), but no interaction time $\times$ group was found $(p=0.622)$. Measured as
A

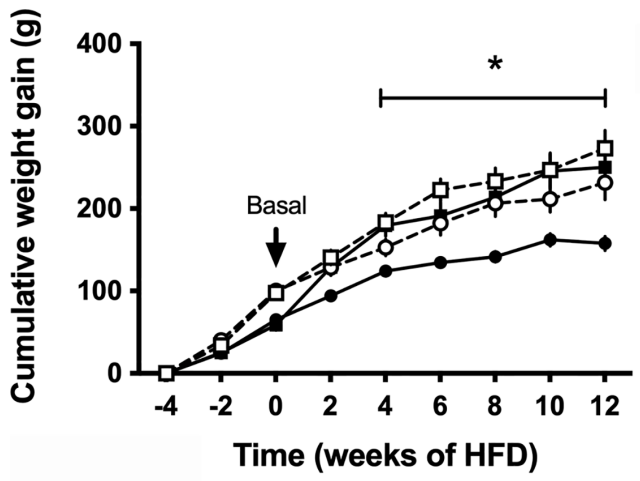

B

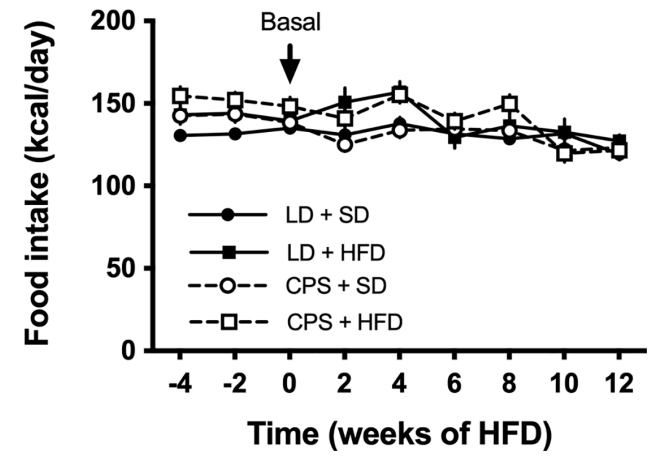

FIGURE 2 | Effects of gestational chronic phase shift of photoperiod on (A) cumulative weight gain and (B) food intake of rats fed with standard diet (SD) and 45\% high-fat diet (HFD) for 12 weeks. Data are mean \pm SEM. Solid line (LD): adult offspring gestated under control photoperiod; dashed line (CPS): adult offspring that had been gestated under chronic photoperiod shift; circles: adult offspring fed with standard diet Prolab ${ }^{\circledR}$ RMH 3000 ; squares: adult offspring fed with HFD. $n=8$ per group. *LD + HFD; CPS + SD and CPS + HFD are different from LD + SD ( $p<0.05$ pairwise comparison at each time point with Bonferroni correction). 
A

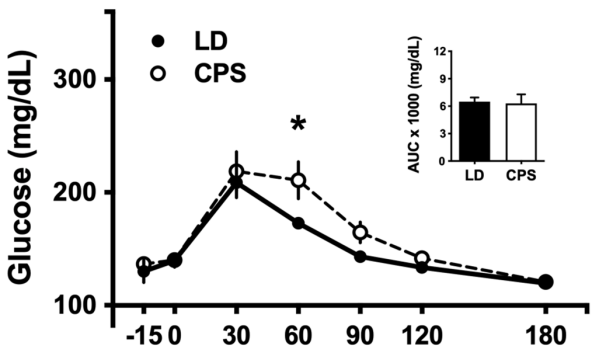

C

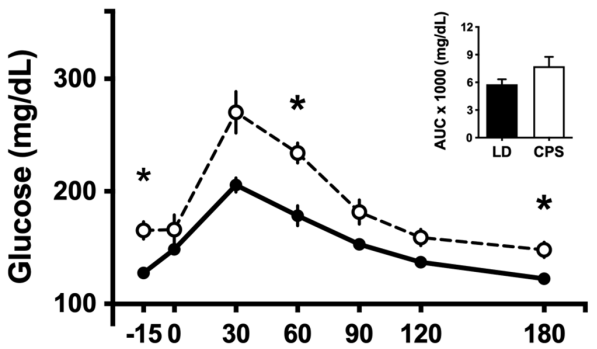

E

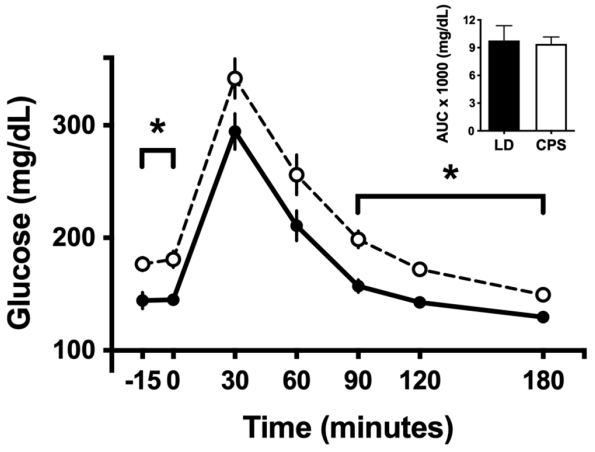

B

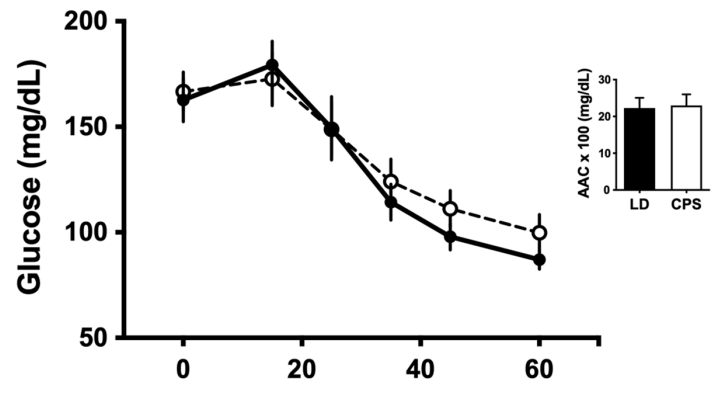

D

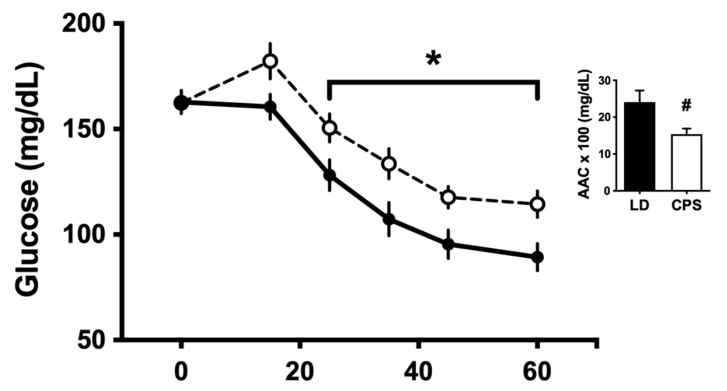

$\mathbf{F}$

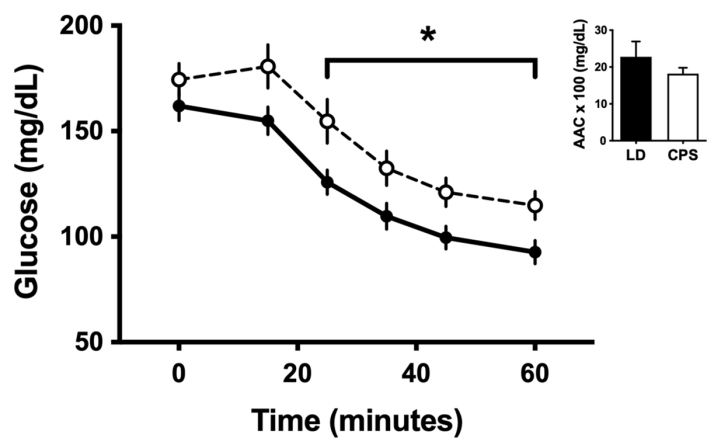

FIGURE 3 | Effects of gestational chronic phase shift of photoperiod on intraperitoneal glucose tolerance test (A, C, E) and intraperitoneal insulin tolerance test (B, D, F) of rats fed with 45\% high-fat diet (HFD) for 12 weeks, measured at week 0/basal (A, B), 6 weeks (C, D) and 12 weeks (E, F). Data are mean \pm SEM. Solid line: LD, adult rats that had been gestated under control 12:12 (LD) photoperiod; dashed line: CPS, adult rats that had been gestated under chronic phase shift (CPS) photoperiod. AUC, Area under the curve; AAC, Area above the curve. $n=8$ per group. *different from LD $(p<0.05$ pairwise comparison at each time point with Bonferroni correction), ${ }^{\#}$ different from LD $(0<0.05$, unpaired $t$-test).

AUC, the glucose response was similar to the CPS group compared to the LD group ( $p=0.838$; unpaired $t$-test), probably due to the fact that the CPS group begins with higher basal plasma levels.

Intraperitoneal insulin tolerance tests were carried out in the parallel cohort 2. One-hundred-day-old animals have shown no difference between LD and CPS offspring (time $\times$ group factor: $p=0.616$, two-way ANOVA; Figure 3B), expressed either as plasma glucose levels or area above the curve (AAC; $p=0.866$ unpaired $t$-test). Similar results were found at 200 days of age (Figure 4B). After 6 weeks of HFD, the group that had been gestated under LD presented a glucose response to insulin alike to the one observed at week 0 (basal). However, the CPS group displayed a marked increase relative to both CPS and LD at week 0 (basal) and to LD at week 6 (time $\times$ group factor: $p=0.026$, two-way ANOVA and Bonferroni; Figure 3D). Glucose response expressed as AAC was at the same level in the LD group regarding week 0 (basal), while CPS showed a significant decrease of AAC ( $p=0.033$; unpaired $t$-test). Finally, a third iITT was performed after 12 weeks under HFD (Figure 3F). No significant increase in glucose response to insulin was found in LD animals regarding week 0 (basal). Meanwhile, CPS animals presented significantly higher levels of glucose in basal conditions, maintaining higher levels of glucose up to the end of the experiment (time $\times$ group factor: $p=0.019$, two-way ANOVA and Bonferroni). However, AAC values for insulin response were lower in the CPS compared to the LD group, but not statistically significant ( $p=0.325$, unpaired $t$-test; Figure $3 \mathbf{F}$ ), probably due to the higher fasting glucose in CPS.

Next, we evaluated the potential role of the endocrine system to help to explain our findings. To this goal, insulin, leptin, and adiponectin plasma levels were measured before applying the 


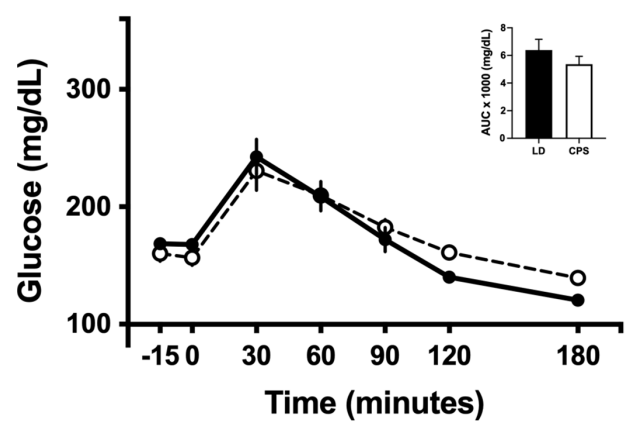

B

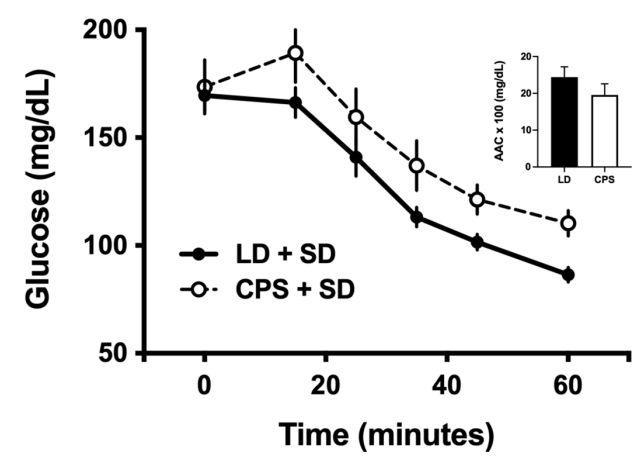

FIGURE 4 | Effects of gestational chronic phase shift of photoperiod on intraperitoneal glucose tolerance test (A) and intraperitoneal insulin tolerance test (B) of rats fed with standard diet (SD) since 100 to 200 days of age. Data are mean \pm SEM. Solid line: LD, adult rats that had been gestated under control 12:12 (LD) photoperiod; dashed line: CPS, adult rats that had been gestated under chronic phase shift (CPS) photoperiod; circles: adult offspring fed with standard diet Prolab ${ }^{\circledR}$ $\mathrm{RMH}$ 3000; squares: adult offspring fed with HFD. $n=8$ per group.

glucose tolerance test in fasting conditions after HFD. First, steady levels of fasting glucose and circulating insulin, leptin, and adiponectin were found throughout 12 weeks under HFD challenge in the control LD group (Figure 5). In contrast, adult CPS offspring displayed increased levels of fasting glucose (time $\times$ group factor: $p=0.025$, two-way ANOVA and
Bonferroni; Figure 5A) at 6 and 12 weeks of treatment. In addition, they exhibited high insulin plasma levels at 6 weeks (time $\times$ group factor: $p=0.016$, two-way ANOVA and Bonferroni; Figure 5B, i e, week 6 versus week 0/basal), dropping to minimal levels after 12 weeks of HFD challenge (i.e., week 12 versus week 6). On the other hand, when plasma
A

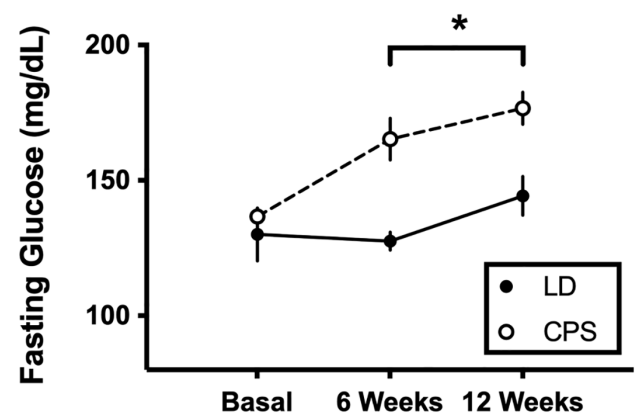

C

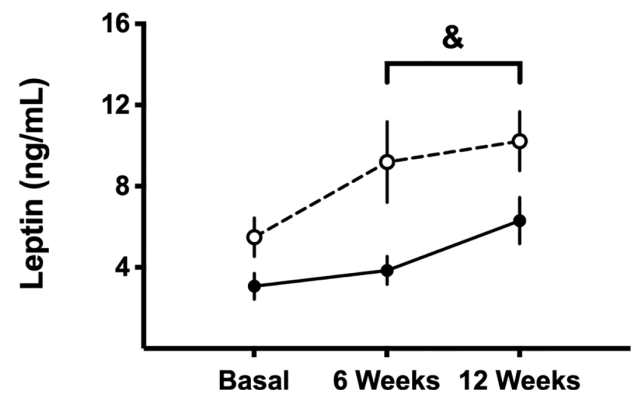

B

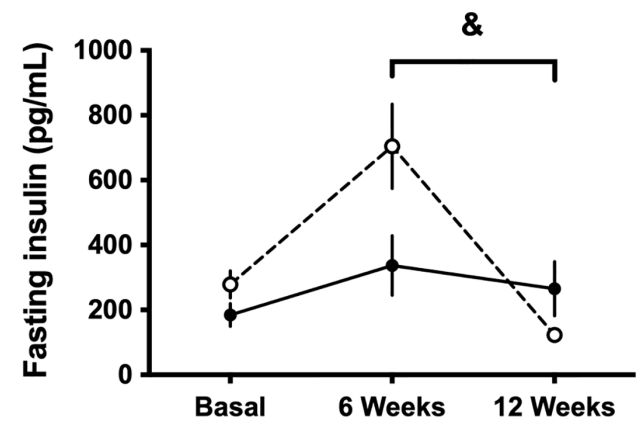

D

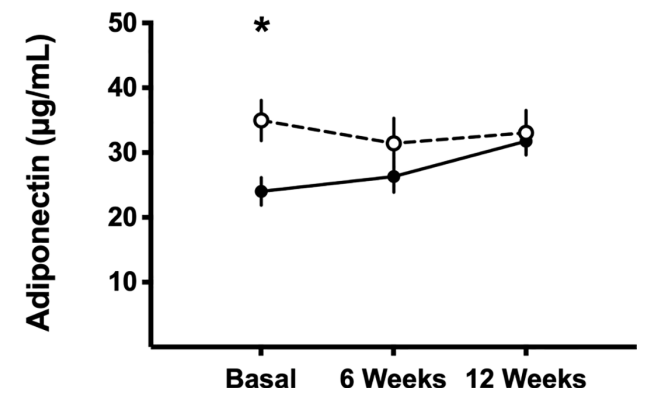

FIGURE 5 | Effects of gestational chronic phase shift of photoperiod on serum levels of fasting glucose (A), fasting insulin (B), leptin (C), and adiponectin (D) at 6 and 12 weeks of $45 \%$ high-fat diet intake. Data are mean \pm SEM. Solid line: LD, adult rats that had been gestated under control 12:12 (LD) photoperiod. Dashed line: CPS, adult rats that had been gestated under chronic phase shift (CPS) photoperiod. $n=8$ per group. *Different from LD $(p<0.05$; two-way ANOVA and Bonferroni test). ${ }^{\&}$ Different from basal ( $p<0.01$; two-way ANOVA and Bonferroni test). 
levels of the two hormones produced by WAT from CPS animals were evaluated, leptin showed constantly increased levels along the 12 weeks under HFD, which is consistent with higher body weight gain (Figure 5C), without an interaction (time $\times$ group factor: $p=0.123$, two-way ANOVA). Adiponectin displayed higher levels already in basal conditions in CPS offspring (i.e., before HFD), but like the levels measured in the control LD offspring, with stable values throughout HFD challenge (time $\times$ group factor: $p=0.149$, two-way ANOVA; Figure 5D).

\section{Effects of Gestational CPS on the Offspring's Adipose Tissue Lipolytic Function In Vitro}

White and brown adipose tissues were collected and analyzed 1 week after the last test described above was carried out. CPS animals fed standard diet had more iWAT and less BAT than animals gestated under control (LD) conditions, while pWAT and eWAT show no difference. HFD induced an increase in perirenal, epigonadal, and inguinal white adipose tissue pads (Figures 6A-C), matching findings from another report (33). Meanwhile, CPS offspring displayed increased subcutaneous iWAT depot with both standard diet and HFD, compared to LD without interaction (column factor: $<0.001$; time $\times$ group factor: $p=0.704$, two-way ANOVA). Surprisingly, CPS iWAT levels under standard diet were similar to those of LD + HFD iWAT. We explored whether the treatments induced an increase in the number of white adipose cells in eWAT. Neither LD nor CPS with or without HFD was associated with differences in the number of cells per section, supporting the idea that the effects induced by HFD or CPS on eWAT total mass represent bigger fat pads. Next, we evaluated the effects of CPS and HFD on brown adipose tissue depot. As expected, HFD given to the control LD offspring did not affect the content of brown adipose tissue (interscapular depot). However, CPS offspring presented a significant decrease (about 50\%; group factor: $p<0.001$, twoway ANOVA and Bonferroni) in iBAT, which was not affected by HFD (time $\times$ group factor: $p=0.697$, two-way ANOVA; (Figure 6D). Neither LD nor CPS with or without HFD displayed differences in the number of cells per section (Figures 6E-H).

The functional integrity of WAT was assessed under in vitro conditions by testing glycerol response to increasing doses of norepinephrine (NE). Explants of LD eWAT animals fed with standard diet had a significant glycerol response to $1 \mu \mathrm{M}$ NE and reached a maximal response with $10 \mu \mathrm{M}$ NE. In contrast, in CPS, eWAT explants' glycerol response to NE was statistically significant only to $10 \mu \mathrm{M} \mathrm{NE}$ (group factor: $p=0.103$, twoway ANOVA and Bonferroni; Figure 7A). In animals fed with HFD, the glycerol response to NE in eWAT LD explants was similar to the one found under the standard diet (group factor: $p=0.64$, treatment $\times$ group factor: $p=0.76$, two-way ANOVA and Bonferroni; Figures 7A-C). Therefore, HFD did not affect the in vitro response to $\mathrm{NE}$ in $\mathrm{LD}$ conditions (Figures $7 \mathrm{C}$ ). In contrast, eWAT explants from adult animals that had been gestated under CPS conditions displayed a blunted glycerol response to all NE doses tested (group factor: $p<0.001$, treatment $\times$ group factor: $p=0.024$, two-way ANOVA and Bonferroni; Figure 7C).

The functionality of BAT was also evaluated under in vitro conditions. First, BAT explants of adult offspring gestated under LD and CPS conditions and fed with standard diet showed increased glycerol response to $10 \mu \mathrm{M}$ NE (group factor: $p=$ 0.029, two-way ANOVA and Bonferroni; Figure 7B). Of note, higher basal levels of glycerol were found in the incubation media from CPS animals than that of control (LD) animals. This difference was no longer present in BAT explants from CPS animals receiving HFD for 12 weeks. In addition, BAT explants from these animals showed a reduced glycerol response to NE and overall glycerol production was lower than that of BAT explants from control (LD) conditions also fed on HFD (group factor: $p<0.001$, treatment $\times$ group factor: $p=0.440$, two-way ANOVA and Bonferroni; Figure 7D).

Some of the differences in white and brown adipose tissue between CPS and LD animals described above were present before the HFD challenge. Therefore, we explored potential epigenetic mechanisms involved by measuring global genomic DNA methylation status in both WAT and BAT depots in the offspring at 100 days. Perirenal WAT from LD offspring showed significant day/night methylation differences, with levels that were about 25\% higher during daytime (Figure 8A). Conversely, lower levels of global genomic DNA methylation were detected during daytime in perirenal WAT from CPS offspring (time $\times$ group factor: $p=0.001$, two-way ANOVA and Bonferroni). It should be noted that the mean day/night methylation level was similar for perirenal WAT from both LD and CPS adult offspring. Finally, no differences were found for global DNA methylation in BAT, either during daytime or nighttime ( $p=$ 0.948, two-way ANOVA; Figure 8B). These early differences in global DNA methylation suggest that epigenetic programming has occurred in WAT from the CPS group.

Next, we evaluated the effects of prenatal chronodisruption and postnatal HFD on total protein expression in white adipose tissue using a quantitative proteomics analysis. (Data are available via ProteomeXchange with identifier PXD026315.)

Animals that had been gestated under LD conditions and received HFD during adult life displayed a significant increase in body weight and weight of white adipose tissue fat pads at 100 days. These exacerbated weight-gain pattern induced by HFD was accompanied by modification in the expression level of 91 proteins (fold change higher than 1.5) in white adipose tissue. We further analyzed the proteomics data using Ingenuity Pathway Analysis (IPA) to look for interactions among the differentially expressed set of proteins framed in functional pathways. As might be expected, the major effects at the proteome level were found in the functional pathway involving fatty acid metabolism. In addition, changes in the expression level of proteins related to cytoskeleton function and cellular differentiation were found.

WAT from adult CPS offspring at 200 days fed with SD displayed differential expression of 275 proteins with a fold change higher than 1.5, when compared to the control LD group fed with SD. Remarkably, 96\% of the differentially 
A
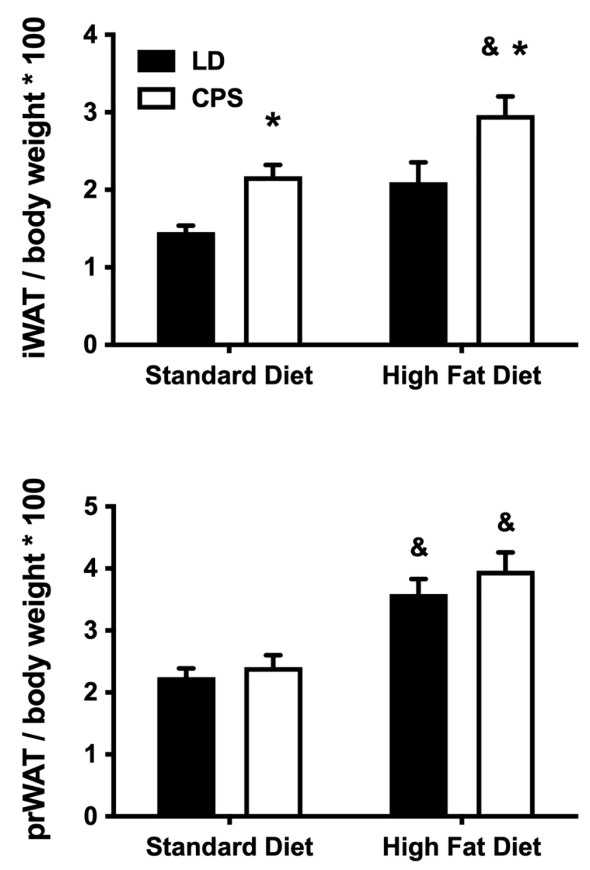

E

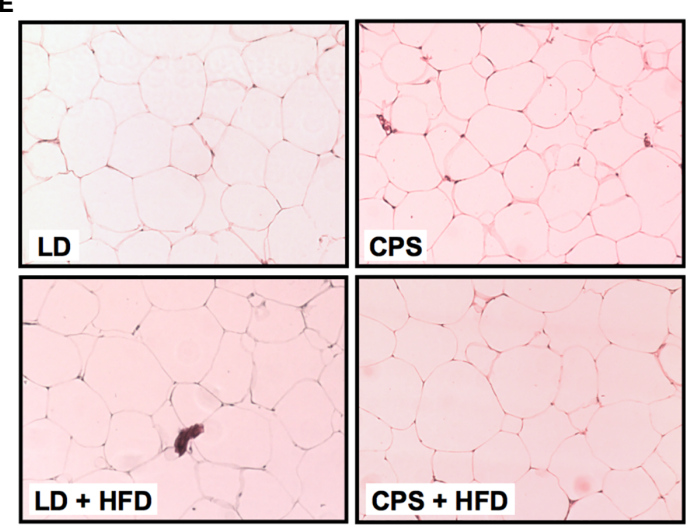

G

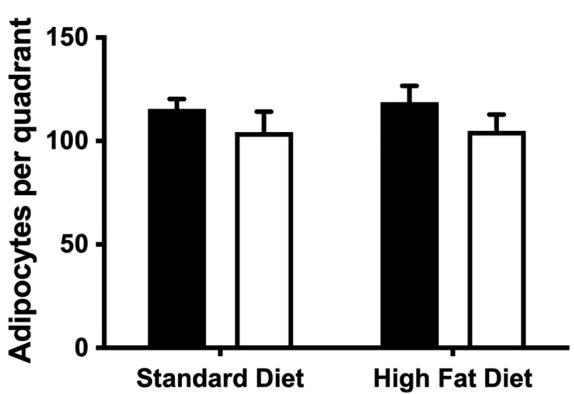

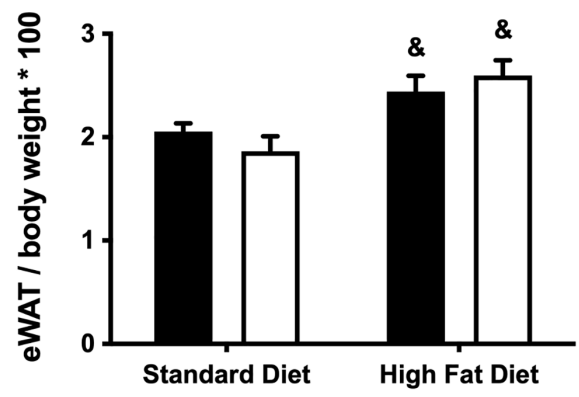

D

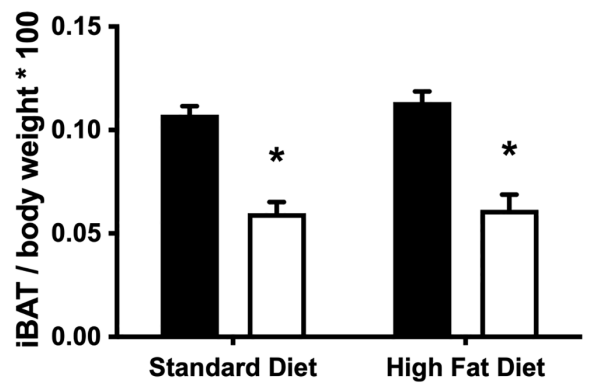

$\mathbf{F}$

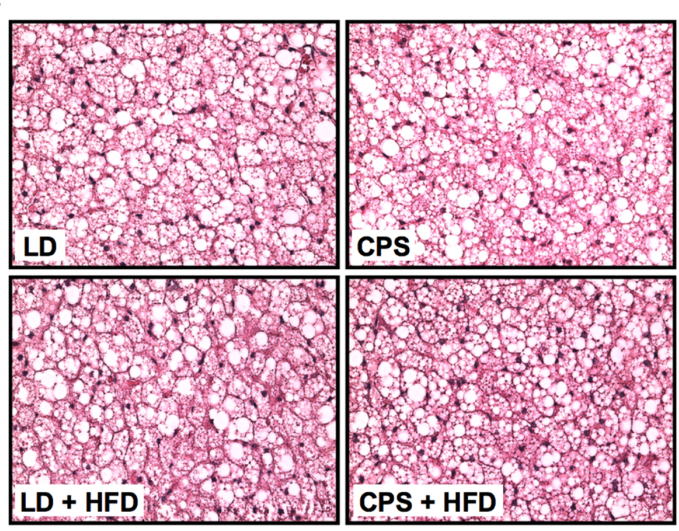

H

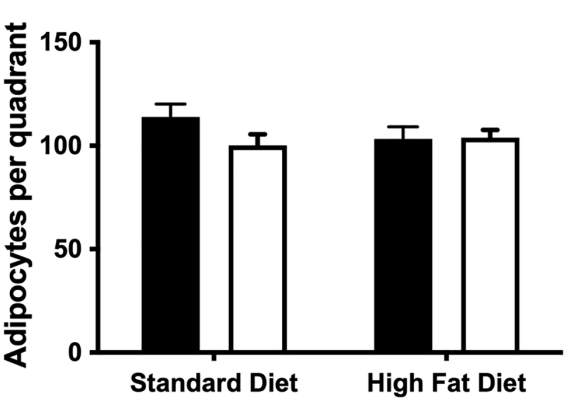

FIGURE 6 | Effects of gestational chronic phase shift of photoperiod in recruitment of adipose tissue depot. Upper panel displays the weight of whole adipose tissue depots adjusted by full-body weight of 200-day-old rats fed with standard diet or high-fat diet. (A) Inguinal adipose tissue, (B) epigonadal adipose tissue, (C) perirenal adipose tissue, (D) interscapular brown adipose tissue. Middle panel displays representative H\&E staining of (E) 10x perirenal white adipose tissue and (F) 40x interscapular adipose tissue histological sections. Lower panel displays the corresponding cell number counts as shown in (G) from white adipose tissue and (H) from brown adipose tissue. Data are mean \pm SEM. Black bars: LD, adult rats that had been gestated under control 12:12 (LD) photoperiod. White bars: CPS, adult rats that had been gestated under chronic phase shift (CPS) photoperiod. Standard diet: animals fed with Prolab ${ }^{\circledR}$ RMH 3000 , High-fat diet: animals fed with $45 \%$ high-fat diet for 12 weeks. $n=8$ per group. *LD + HFD; CPS + SD and CPS + HFD are different from LD + SD ( $p<0.05$ pairwise comparison at each time point with Bonferroni correction). ${ }^{8}$ Different from standard diet $(p<0.05$ pairwise comparison at each time point with Bonferroni correction). 


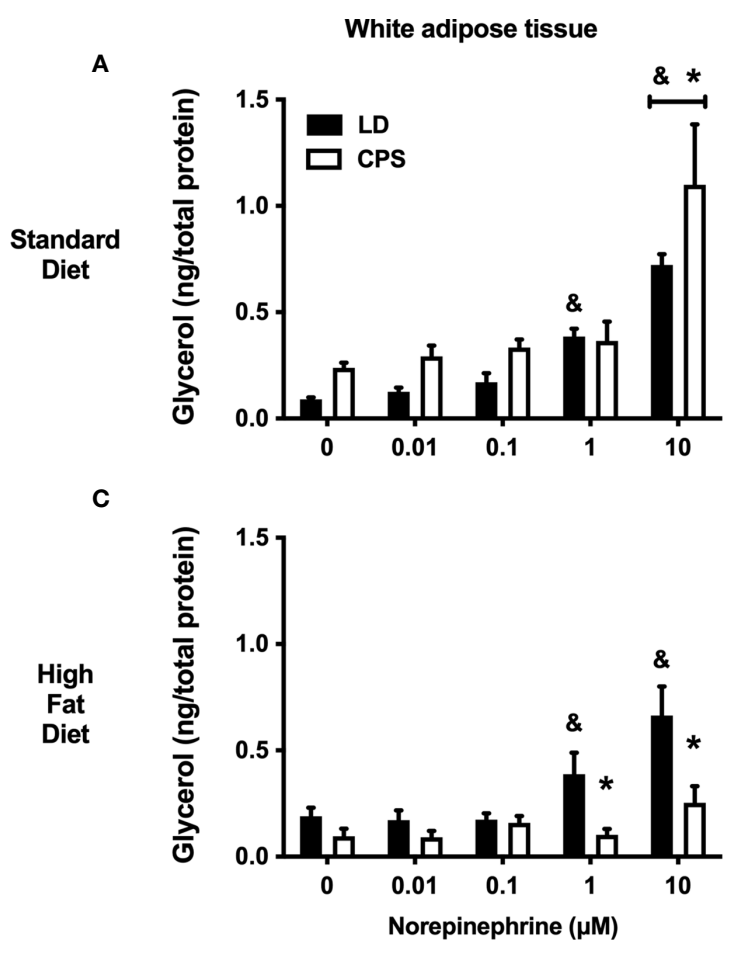

B

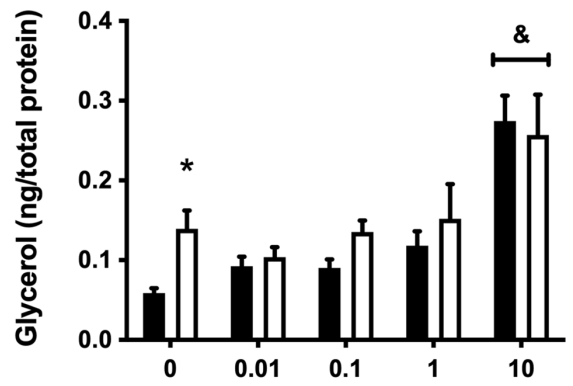

D

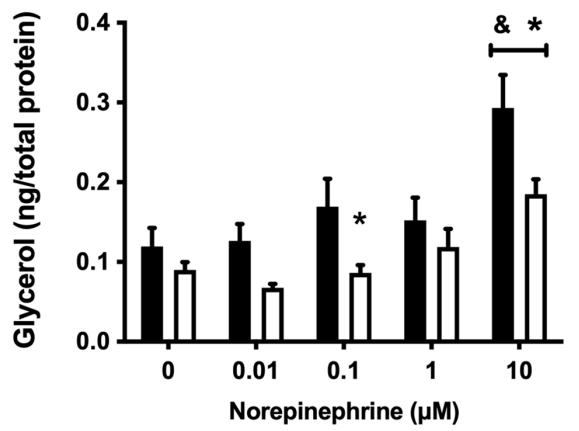

FIGURE 7 | Effects of gestational chronic phase shift of photoperiod on glycerol response to increasing doses of norepinephrine (NE) of white epigonadal adipose tissue (A, C) and brown interscapular adipose tissue (B, D). Explants from 200-day-old rats fed with either standard diet Prolab ${ }^{\circledR} \mathrm{RMH} 3000$ (A, B) or $45 \%$ high-fat diet (HFD; C, D). Data are mean \pm SEM. Black bars: LD, adult rats that had been gestated under control $12: 12$ (LD) photoperiod. White bars: CPS, adult rats that had been gestated under chronic phase shift (CPS) photoperiod. $n=8$ per group. *different from LD. ${ }^{\star}$ Different from basal LD (0 $\mu M$ NE). All comparisons: $p<0.05$ pairwise comparison at each time point with Bonferroni correction.

expressed proteins were downregulated in WAT from adult CPS offspring. This significant proportion of downregulated proteins is consistent with the metabolic alterations described above for WAT from adult CPS relative to LD offspring.

Comparison of adult CPS offspring fed standard versus CPS fed HFD led to the identification of 235 proteins exhibiting significantly modified expression levels, that is to say, 114 more proteins than LD animals receiving HFD. Among these, 219 proteins were upregulated and 16 proteins were downregulated.

In contrast, comparison of WAT from CPS fed with SD and LD fed with HFD vs. both LD fed with SD show striking similarity and only four proteins were differentially expressed

\section{White adipose tissue}

A

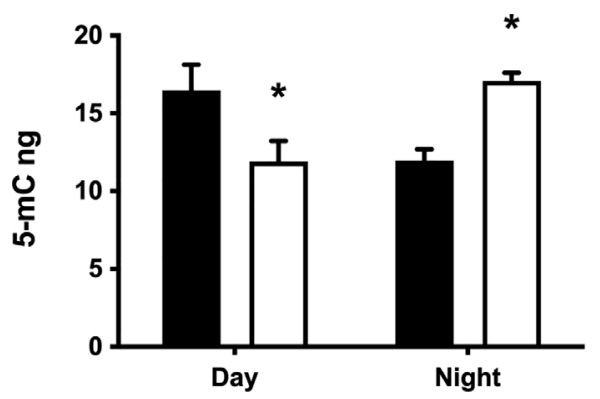

Brown adipose tissue

B

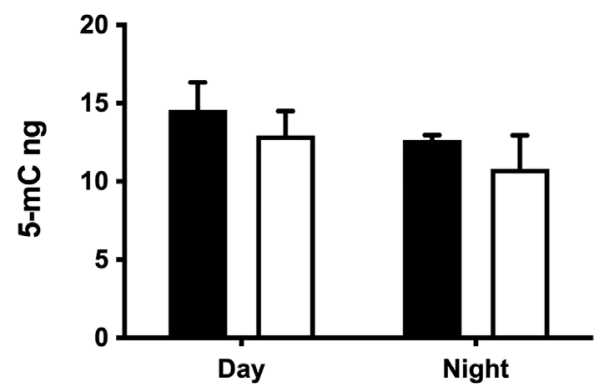

FIGURE 8 | Effects of gestational chronic phase shift of photoperiod on global genomic DNA methylation of perirenal white adipose tissue (A) and interscapular brown adipose tissue (B) from 90-day-old rats sampled at day (10:00 h) and night (22:00 h). Black bars: LD, adult rats that had been gestated under control 12:12 (LD) photoperiod. White bars: CPS, adult rats that had been gestated under chronic phase shift (CPS) photoperiod. $n=5$ per group. ${ }^{*}$ different from LD $(p<0.05$ pairwise comparison at each time point with Bonferroni correction). 
in CPS HFD. Taken together, these proteomics results support the notion that in terms of protein expression, WAT from adult CPS offspring is somewhat similar to WAT from adult LD offspring fed with HFD. This possibility was further investigated by running functional proteomic analyses to target common protein profiles by these means; 20 differentially expressed proteins were identified in both experimental groups as compared to the adult offspring that had been gestated under control LD conditions receiving SD postnatally (Figure 9). Of note, a most important difference in WAT was found in MYADM, that was -9.7 times downregulated regarding LD + HFD. So far, the actual role of MYADM in adipose tissue function remains non-investigated, but recent evidence is in line with a potential role of this protein in local inflammation, as mediator of low-grade inflammation as deduced for its role in membrane (34). After this exploratory analysis, we decided to focus on the common proteins for the CPS and LD + HFD groups, based on our observed results. Under this criterion, we selected the 20 proteins shown in Figure 9, grouped to build a new dataset and analyzed by IPA to generate the interactomes.

Detailed pathway analysis of WAT from adult CPS + SD showed an imbalance in the pathways related to inflammatory status, network 1 (TNF/IL4, score 14; Figure 10, top panel), and network 2 (AKT/ERK, score 28; Figure 10, lower panel) supporting the idea that WAT from adult CPS offspring fed with SD was undoubtedly affected to an important extent by gestational chronodisruption.

\section{DISCUSSION}

Life on Earth has evolved over million years to successfully adapt to dominant environmental clues such as light/dark cycles. However, several environmental factors have been influenced by human activity, with artificial light at night (ALAN) being one of the most recent changes imposed over roughly the last century. ALAN is linked not only to light pollution of densely populated locations but also to shift work schedules (about $20 \%$ of the global workforce). Recent research on the crosstalk between metabolism and the circadian system has provided evidence linking shift work schedules with the onset of metabolic disorders such as obesity, type 2 diabetes, and cardiovascular dysfunction (35). Considering that a clear

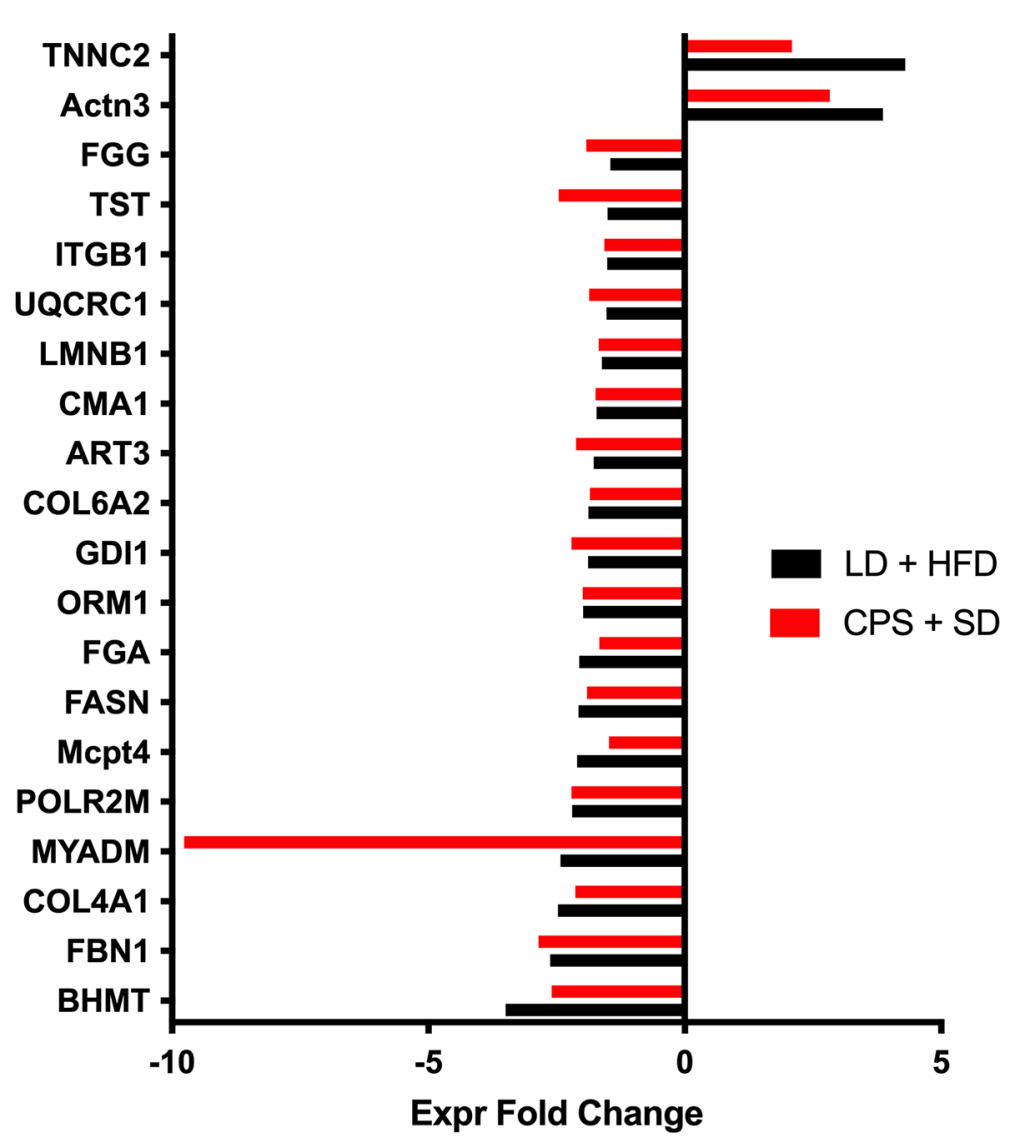

FIGURE 9 | Up and down expressed proteins in WAT from adult offspring gestated under control conditions and fed with high-fat diet during 12 weeks from postnatal day 100 onwards (LD + HFD group - black bar) and adult offspring gestated under CPS conditions and fed with standard diet postnatally (CPS + SD). Fold change in CPS + SD offspring is shown in red, and that of LD + HFD is in black. Data are expressed as mean of fold changes and were analyzed using Ingenuity Pathways Analysis (IPA). 


\begin{tabular}{|l|r|r|}
\hline Gene Name & LD + HFD & CPS + SD \\
\hline COL6A2 & $-1,878$ & $-1,851$ \\
\hline FGA & $-2,057$ & $-1,663$ \\
\hline FGG & $-1,453$ & $-1,922$ \\
\hline GDI1 & $-1,886$ & $-2,211$ \\
\hline ORM1 & $-1,98$ & $-1,988$ \\
\hline TNNC2 & 4,297 & 2,088 \\
\hline
\end{tabular}
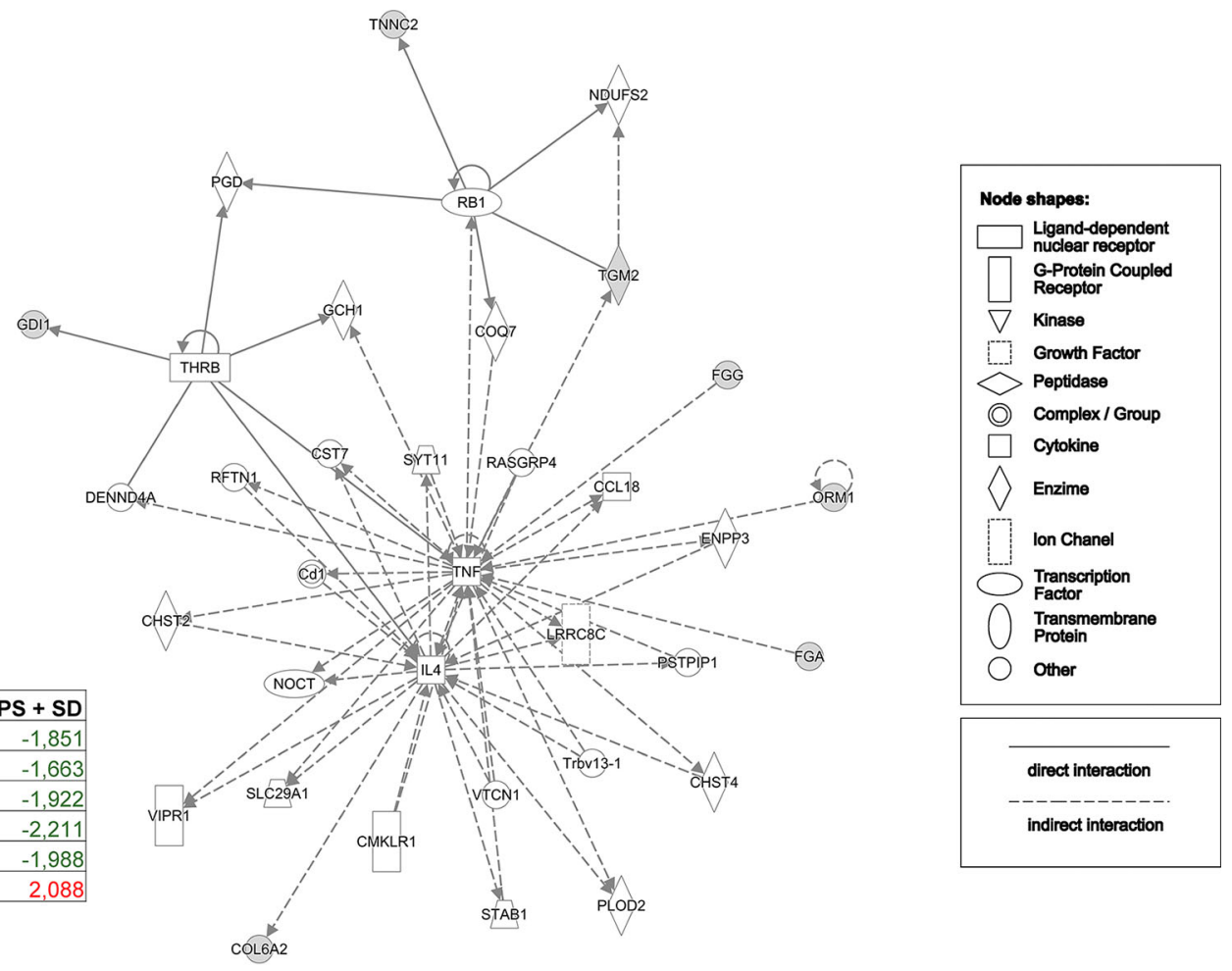

\begin{tabular}{|l|r|r|}
\hline Gene Name & LD + HFD & CPS + SD \\
\hline Actn3 & 3,862 & 2,826 \\
\hline BHMT & $-3,497$ & $-2,596$ \\
\hline CMA1 & $-1,722$ & $-1,742$ \\
\hline COL4A1 & $-2,471$ & $-2,134$ \\
\hline FASN & $-2,071$ & $-1,903$ \\
\hline FBN1 & $-2,622$ & $-2,849$ \\
\hline ITGB1 & $-1,514$ & $-1,565$ \\
\hline LMNB1 & $-1,613$ & $-1,677$ \\
\hline MCPt4 & $-2,097$ & $-1,481$ \\
\hline MYADM & $-2,427$ & $-9,76$ \\
\hline TST & $-1,509$ & $-2,46$ \\
\hline UQCRC1 & $-1,53$ & $-1,868$ \\
\hline
\end{tabular}

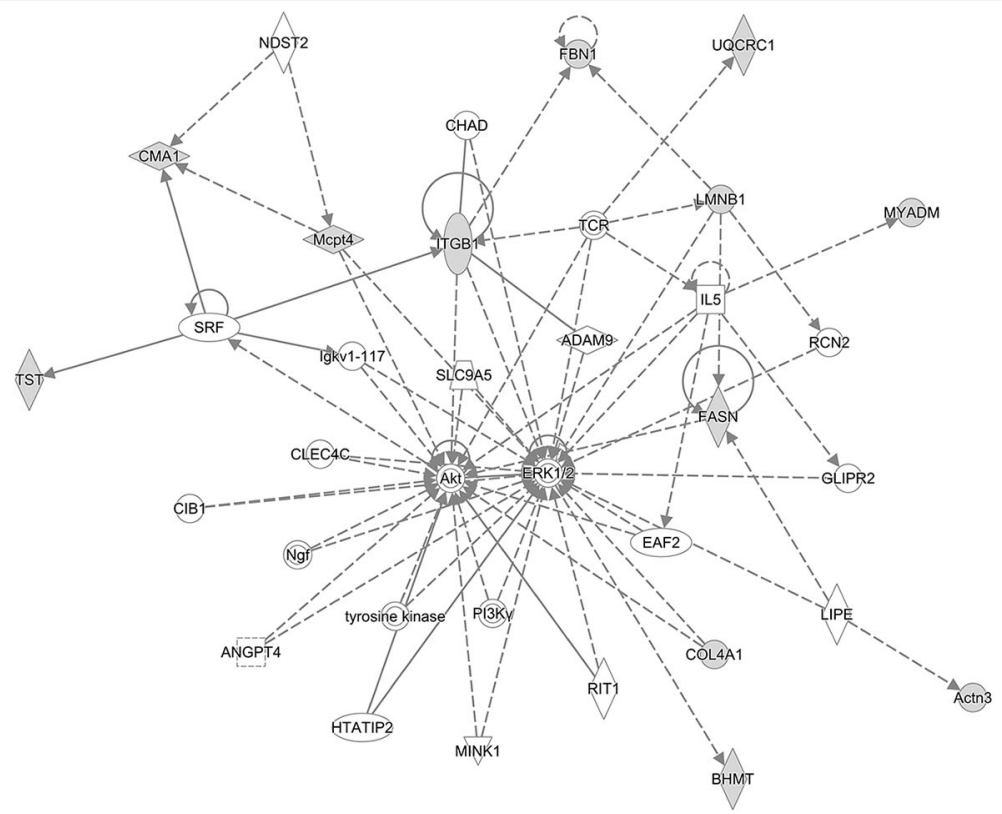

FIGURE 10 | Interactome depicting the pathways that were most significantly enriched by differentially expressed proteins identified by quantitative proteomics of white adipose tissue (WAT) from adult offspring. Comparison: adult offspring that had been gestated under control (LD) conditions and fed with high-fat diet (HFD) during 12 weeks from postnatal day 100 onwards (LD + HFD group) versus adult offspring that had been gestated under CPS conditions and fed with standard diet postnatally (CPS + SD). Red, upregulated protein; green, downregulated protein; white, protein related in the network without expression changes. Proteins connected with solid lines have direct links; those connected with dotted lines have indirect links. The set of proteins was grouped in the Ingenuity Top Network 1 : Cellular Development, Cell Morphology, Cell-Mediated Immune Response, and Inflammatory Response pathways. 
relationship has been established for an adverse intrauterine milieu with chronic disease later in life [references in $(36,37)]$, we tested the hypothesis that gestational chronodisruption (CPS conditions) translates into long-term detrimental effects on glucose homeostasis and adipose tissue physiology, increasing the negative impact of HFD as a cardiometabolic challenge during adulthood in the male offspring.

Gestation under a CPS has an important effect on the adrenal and cardiovascular system in the male offspring during adulthood $(6,7,30)$. In the present paper, we found the impact of CPS on adipose tissue recruitment and function under standard diet that were enhanced by the HFD challenge. At 100 days of age, several differences were present between the LD and CPS group. We observed significant differences in intraperitoneal glucose tolerance test, requiring more time to return to the basal levels, accompanied by an increase in leptin, suggesting an increase of adipose tissue that may be involved in the early metabolic effect described above. In addition, animals gestated under CPS conditions responded to HFD with altered iGTT and iITT profiles, in parallel with increased weight gain without any change in food intake. In keeping with early CPS effects on adipose tissue, at 100 days of age in WAT perirenal, global methylation displays differences between LD and CPS, with CPS offspring showing lower levels of global genomic DNA methylation during daytime. Altogether, the current results, before the challenge using HFD, support the idea that an epigenetic mechanism should be present in the early effect observed in WAT at 100 days of age. Next, we found that chronodisruption throughout gestation induced significant changes in body weight and adipose tissue recruitment and function under standard diet. CPS effects on adipose tissue were exacerbated by HFD. By 200 days of age and after 12 weeks of receiving HFD, the distribution of different adipose tissue depots was altered. Besides, the amount of inguinal white adipose tissue (iWAT) was increased in the adult CPS offspring, while the amount of interscapular brown adipose tissue (iBAT) decreased significantly. Furthermore, the in vitro lipolytic capacity of adipose tissue from CPS offspring (evaluated by glycerol production) was inhibited in animals exposed to prenatal CPS and postnatal HFD. At 200 days old (after 12 weeks with HFD), an obesogenic phenotype was observed in CPS animals, which, at the molecular level, might be explained by the altered expression pattern found for 275 proteins in CPS adipose tissue, as compared with control (LD) animals fed with a standard diet. Interestingly, the CPS $+\mathrm{SD}$ and LD + HFD groups showed 20 differentially expressed proteins in common ( 2 upregulated and 18 downregulated). Based on these common proteins, functional analyses identified two functional pathways as significantly altered: network 1-TNF/IL4 and network 2-AKT/ ERK. Altogether, the present results support the notion that white adipose tissue function was programmed in utero by chronodisruption, inducing changes in physiology and metabolic response capacity that persist into adulthood. Interestingly, the key results in the current manuscript support the idea that CPS offspring treated with a standard diet, in terms of metabolic assessment, are close to a control animal fed with a HFD.

Increased accumulation of white adipose tissue has been proposed as a strong and independent predictor of adverse health outcomes associated with obesity (38), as reported here. Besides its role in energy storage, WAT is also an active secretory organ producing a large number of molecules termed adipokines. Actually, adipokines participate in the modulation of glucose and lipid homeostasis via the central effects of leptin (39). Adipokines include pro-inflammatory factors and chemokines, increased in obesity (40). Although obesity has been associated with increased accumulation of macrophages within the fat mass, it remains unclear how the crosstalk between macrophages and white adipocyte tissue initiates the dysfunction of white adipose tissue in metabolic disorders. Recently, it has been demonstrated that a link between metabolic syndrome and chronodisruption is provided by the early onset of low-grade inflammation $(38,41)$, an inflammatory state associated with a wide range of chronic conditions, such as metabolic syndrome, type 2 diabetes mellitus, and cardiovascular disease (42). Reinforcing this issue, our current results plus other works of our group show that maternal chronodisruption is translated in the offspring in an obesogenic phenotype in which low-grade inflammation is already present at 90 days of age (30); it must be kept in mind that these findings are consistent with the significant range of differentially expressed proteins identified by means of quantitative proteomics in WAT.

We suggest that chronodisruption interfered with the normal developmental trajectory of adipose tissue in utero, which led to readily consequences for metabolism during adult life. The persistent deleterious effects secondary to light at night along gestation suggest the involvement of epigenetic mechanisms. Indeed, a significant change in the levels of global genomic DNA methylation between day and night was detected in white adipose tissue. On the other hand, in vitro glycerol production in response to norepinephrine was affected. Therefore, regulatory mechanisms related to the misalignment of circadian rhythms may operate in WAT. In this context, it is worth mentioning that in a recent publication, Moreno-Mendez et al. (43) argued that epigenetic impairment of adipose tissue function might derive from a mechanism involving hypomethylation of IGF2 and hypermethylation of leptin and TNF-A. This is consistent with our findings that independent of the diet, animals exposed to chronodisruption during gestation between 100 and 200 days of age are metabolically comparable to those LD animals fed with HFD. A series of cases reported by Nahme et al. (44) in 2019 suggest that these findings can be extrapolated to human health. These authors, for the first time, observed a direct relationship between misalignment of the melatonin rhythm during pregnancy and gestational problems together with a low Apgar score in the newborns from these mothers. While we must be careful to overinterpret a series of cases, there are already many studies suggesting an increased risk of adverse pregnancy outcomes secondary to maternal shift work throughout pregnancy (12) and in their offspring $(45,46)$. 
For instance, it has been demonstrated that deficient nutrition during gestation alters the development of adipocytes in utero, resulting in a permanent increase in the ability to form new adipocytes in adipose tissue depots and increase lipid storage in existing adipocytes (47).

In addition, we know that maternal melatonin during pregnancy may also play an important role in the development of brown adipose tissue and the thermoregulation of the newborn sheep $(48,49)$. Circadian disruption chronically impairs the biological clock's function, favoring multiple pathological processes like cancer and metabolic and cardiovascular disorders (50). Many of these processes could be related to a low-grade inflammation state, as suggested by the upregulation of TNF-a, IL-1b, and IL-6 in various tissues from rat and other animal models of chronodisruption in sleep deprivation $(51,52)$. Of note, our proteomic results suggest changes in the same direction. Low-grade inflammation has been implicated in the development of chronic diseases. For instance, undernutrition in utero causes impairment in muscle growth during fetal growth, and after birth, these individuals accumulate a disproportionately high-fat mass (53). The adipose tissue secretes several potent inflammatory factors, which may lead to low-grade inflammation. Though there are scarce evidence that links gestational chronodisruption with a lowgrade inflammation state, our results suggest that gestational chronodisruption promotes an increase of pro-inflammatory cytokines in males (30), which could be the central mechanism of programming observed here. Consistent with this evidence, we observed that two pivotal pathways involved in adipose tissue function like TNF/IL4 and AKT/ERK were modified in LD fed with HFD and CPS with standard diet at 200 days of age; both pathways involved a key step in the progression of metabolic adipose tissue dysfunction like low-grade inflammation and differentiation process (54). Therefore, we speculate that part of this key pathways was modified in utero and could be one of the mechanisms programming in utero by gestational chronodisruption, an idea that needs more exploration. In the same line, results of our group support the notion that a critical organ involved in metabolism and correct response to stress, the adrenal gland, is programmed in utero by maternal chronodisruption, in which CPS offspring present a desynchronization of the adrenal circadian clock and steroidogenic pathway, leading to abnormal stress responses and potentially increasing the risk of developing chronic diseases $(6,7)$.

An interesting comparison of our results with those available in sheep suggests to us that sheep are more resilient than the rat to the impact of photoperiod changes during gestation $(21,55)$. The evidence supports those minor changes that occur in pregnant sheep in comparison to the pregnant rat as well as the minor changes observed in the young lamb offspring, opening the possibility to speculate that a protective physiological environment allows the sheep to cope with adverse environmental cues. How this occurs remains to be investigated, but we need to keep in mind that the sheep present several physiological differences compared to rats, such as glucocorticoid production, which is low in sheep (56), pregnancy physiology, and the seasonal characteristic of the sheep and postnatal development. Further research is needed in order to establish how both animal models help to understand the long-term impact of gestational chronodisruption in humans.

In summary, the present results in male rats strongly support the idea that gestational chronodisruption may act through epigenetic mechanisms to define an abnormal adipose tissue phenotype, which is functionally consistent with increased risk of obesity, insulin resistance, hypertension, and metabolic syndrome. This liability seems to be compounded by HFD, which can be considered a highly prevalent risk factor in modern life.

\section{DATA AVAILABILITY STATEMENT}

The raw data supporting the conclusions of this article will be made available by the authors, without undue reservation. Protein data are available via ProteomeXchange with identifier PXD026315 https://www.ebi.ac.uk/pride/archive/projects/PXD026315.

\section{ETHICS STATEMENT}

The animal study was reviewed and approved by Bioethics Commission from the Universidad Austral de Chile (CBA: 352/2019).

\section{AUTHOR CONTRIBUTIONS}

DH, CT-F, and HR conceived and designed the study, analyzed and interpreted the data, drafted the manuscript, critically revised important intellectual content in the manuscript, and provided overall supervision. NM, TK, CS, ES, KV, FT, and MS-F performed the experiments, analyzed the data, drafted the manuscript, and contributed to intellectual content in the manuscript. All authors contributed to the article and approved the submitted version.

\section{FUNDING}

This work was supported by Grants Fondecyt 11190711 (DH), Fondecyt 1191207 (CT-F), and Fondecyt 11170245 (NM) from Fondo Nacional de Desarrollo Científico y Tecnológico, Chile.

\section{SUPPLEMENTARY MATERIAL}

The Supplementary Material for this article can be found online at: https://www.frontiersin.org/articles/10.3389/fendo.2021.678468/ full\#supplementary-material 


\section{REFERENCES}

1. U.S. Department of Labor B of LS. Workers on Flexible and Shift Schedules in May 2004. BLS News (2005).

2. Parent-Thirion A. European Foundation for the Improvement of Living and Working Conditions Eds. In: Fourth European Working Conditions Survey. Luxembourg: Office for Offical Publ. of the European Communities [u.a.] (2007).

3. Knutsson A. Health Disorders of Shift Workers. Occup Med (2003) 53:103-8. doi: 10.1093/occmed/kqg048

4. Jones ME, Schoemaker MJ, McFadden EC, Wright LB, Johns LE, Swerdlow AJ. Night Shift Work and Risk of Breast Cancer in Women: The Generations Study Cohort. Br J Cancer (2019) 121:172-9. doi: 10.1038/s41416-019-0485-7

5. Strohmaier S, Devore EE, Huang T, Vetter C, Eliassen AH, Rosner B, et al. Maternal Rotating Night Shift Work Before Pregnancy and Offspring Stress Markers. Physiol Behav (2019) 207:185-93. doi: 10.1016/j.physbeh.2019.05.007

6. Mendez N, Halabi D, Spichiger C, Salazar ER, Vergara K, Alonso-Vasquez P, et al. Gestational Chronodisruption Impairs Circadian Physiology in Rat Male Offspring, Increasing the Risk of Chronic Disease. Endocrinology (2016) 157:4654-68. doi: 10.1210/en.2016-1282

7. Salazar ER, Richter HG, Spichiger C, Mendez N, Halabi D, Vergara K, et al. Gestational Chronodisruption Leads to Persistent Changes in the Rat Fetal and Adult Adrenal Clock and Function: Fetal Programming of Adrenal Dysfunction by Photoperiod Shifting. J Physiol (2018) 596:5839-57. doi: 10.1113/JP276083

8. Galdames HA, Torres-Farfan C, Spichiger C, Mendez N, Abarzua-Catalan L, Alonso-Vazquez P, et al. Impact of Gestational Chronodisruption on Fetal Cardiac Genomics. J Mol Cell Cardiol (2014) 66:1-11. doi: 10.1016/ j.yjmcc.2013.10.020

9. Varcoe TJ, Wight N, Voultsios A, Salkeld MD, Kennaway DJ. Chronic Phase Shifts of the Photoperiod Throughout Pregnancy Programs Glucose Intolerance and Insulin Resistance in the Rat. PloS One (2011) 6:e18504. doi: 10.1371/journal.pone.0018504

10. Lin Y-C, Chen M-H, Hsieh C-J, Chen P-C. Effect of Rotating Shift Work on Childbearing and Birth Weight: A Study of Women Working in a Semiconductor Manufacturing Factory. World J Pediatr (2011) 7:129-35. doi: 10.1007/s12519-011-0265-9

11. Reschke L, McCarthy R, Herzog ED, Fay JC, Jungheim ES, England SK. Chronodisruption: An Untimely Cause of Preterm Birth? Best Pract Res Clin Obstetrics Gynaecol (2018) 52:60-7. doi: 10.1016/j.bpobgyn.2018.08.001

12. Cai C, Vandermeer B, Khurana R, Nerenberg K, Featherstone R, Sebastianski $\mathrm{M}$, et al. The Impact of Occupational Shift Work and Working Hours During Pregnancy on Health Outcomes: A Systematic Review and Meta-Analysis. Am J Obstetrics Gynecol (2019) 221:563-76. doi: 10.1016/j.ajog.2019.06.051

13. Strohmaier S, Devore EE, Vetter C, Missmer S, Heather Eliassen A, Rosner B, et al. Night Shift Work Before and During Pregnancy and Offspring Weight Outcomes Through Adolescence. Obesity (2018) 26:1491-500. doi: 10.1002/ oby. 22267

14. Strohmaier S, Devore EE, Vetter C, Eliassen AH, Rosner B, Okereke OI, et al. Night Shift Work Before and During Pregnancy in Relation to Depression and Anxiety in Adolescent and Young Adult Offspring. Eur J Epidemiol (2019) 34:625-35. doi: 10.1007/s10654-019-00525-2

15. Vilches N, Spichiger C, Mendez N, Abarzua-Catalan L, Galdames HA, Hazlerigg DG, et al. Gestational Chronodisruption Impairs Hippocampal Expression of NMDA Receptor Subunits Grin1b/Grin3a and Spatial Memory in the Adult Offspring. PloS One (2014) 9:e91313. doi: 10.1371/ journal.pone.0091313

16. Ferreira DS, Amaral FG, Mesquita CC, Barbosa APL, Lellis-Santos C, Turati $\mathrm{AO}$, et al. Maternal Melatonin Programs the Daily Pattern of Energy Metabolism in Adult Offspring. PloS One (2012) 7:e38795. doi: 10.1371/ journal.pone.0038795

17. Spichiger C, Torres-Farfan C, Galdames HA, Mendez N, Alonso-Vazquez P, Richter HG. Gestation Under Chronic Constant Light Leads to Extensive Gene Expression Changes in the Fetal Rat Liver. Physiol Genomics (2015) 47:621-33. doi: 10.1152/physiolgenomics.00023.2015

18. Nogueira TC, Lellis-Santos C, Jesus DS, Taneda M, Rodrigues SC, Amaral FG, et al. Absence of Melatonin Induces Night-Time Hepatic Insulin Resistance and Increased Gluconeogenesis Due to Stimulation of Nocturnal Unfolded
Protein Response. Endocrinology (2011) 152:1253-63. doi: 10.1210/en.20101088

19. Torres-Farfan C, Rocco V, Monsó C, Valenzuela FJ, Campino C, Germain A, et al. Maternal Melatonin Effects on Clock Gene Expression in a Nonhuman Primate Fetus. Endocrinology (2006) 147:4618-26. doi: 10.1210/en.2006-0628

20. Varcoe TJ, Gatford KL, Kennaway DJ. Maternal Circadian Rhythms and the Programming of Adult Health and Disease. Am J Physiol-Regulatory Integr Comp Physiol (2018) 314:R231-41. doi: 10.1152/ajpregu.00248.2017

21. Gatford KL, Kennaway DJ, Liu H, Schultz CG, Wooldridge AL, Kuchel TR, et al. Simulated Shift Work During Pregnancy Does Not Impair Progeny Metabolic Outcomes in Sheep. J Physiol (2020) 598:5807-19. doi: 10.1113/ JP280341

22. Bellou V, Belbasis L, Tzoulaki I, Evangelou E. Risk Factors for Type 2 Diabetes Mellitus: An Exposure-Wide Umbrella Review of Meta-Analyses. PloS One (2018) 13:e0194127. doi: 10.1371/journal.pone.0194127

23. Jayedi A, Rashidy-Pour A, Khorshidi M, Shab-Bidar S. Body Mass Index, Abdominal Adiposity, Weight Gain and Risk of Developing Hypertension: A Systematic Review and Dose-Response Meta-Analysis of More Than 2.3 Million Participants: Adiposity and Risk of Hypertensione. Obes Rev (2018) 19:654-67. doi: 10.1111/obr.12656

24. Kivimäki M, Kuosma E, Ferrie JE, Luukkonen R, Nyberg ST, Alfredsson L, et al. Overweight, Obesity, and Risk of Cardiometabolic Multimorbidity: Pooled Analysis of Individual-Level Data for 120813 Adults From 16 Cohort Studies From the USA and Europe. Lancet Public Health (2017) 2: e277-85. doi: 10.1016/S2468-2667(17)30074-9

25. Bhaskaran K, Douglas I, Forbes H, dos-Santos-Silva I, Leon DA, Smeeth L. Body-Mass Index and Risk of 22 Specific Cancers: A Population-Based Cohort Study of 5.24 Million UK Adults. Lancet (2014) 384:755-65. doi: 10.1016/ S0140-6736(14)60892-8

26. Reilly SM, Saltiel AR. Adapting to Obesity With Adipose Tissue Inflammation. Nat Rev Endocrinol (2017) 13:633-43. doi: 10.1038/ nrendo.2017.90

27. Nedergaard J, Golozoubova V, Matthias A, Asadi A, Jacobsson A, Cannon B. UCP1: The Only Protein Able to Mediate Adaptive non-Shivering Thermogenesis and Metabolic Inefficiency. Biochim Biophys Acta (2001) 1504:82-106. doi: 10.1016/S0005-2728(00)00247-4

28. Saito M, Okamatsu-Ogura Y, Matsushita M, Watanabe K, Yoneshiro T, NioKobayashi J, et al. High Incidence of Metabolically Active Brown Adipose Tissue in Healthy Adult Humans: Effects of Cold Exposure and Adiposity. Diabetes (2009) 58:1526-31. doi: 10.2337/db09-0530

29. Dearden L, Bouret SG, Ozanne SE. Sex and Gender Differences in Developmental Programming of Metabolism. Mol Metab (2018) 15:8-19. doi: 10.1016/j.molmet.2018.04.007

30. Mendez N, Torres-Farfan C, Salazar E, Bascur P, Bastidas C, Vergara K, et al. Fetal Programming of Renal Dysfunction and High Blood Pressure by Chronodisruption. Front Endocrinol (2019) 10:362. doi: 10.3389/ fendo.2019.00362

31. Perez-Riverol Y, Csordas A, Bai J, Bernal-Llinares M, Hewapathirana S, Kundu DJ, et al. The PRIDE Database and Related Tools and Resources in 2019: Improving Support for Quantification Data. Nucleic Acids Res (2019) 47: D442-50. doi: 10.1093/nar/gky1106

32. Wei J, Carroll RJ, Harden KK, Wu G. Comparisons of Treatment Means When Factors Do Not Interact in Two-Factorial Studies. Amino Acids (2012) 42:2031-5. doi: 10.1007/s00726-011-0924-0

33. Marques C, Meireles M, Norberto S, Leite J, Freitas J, Pestana D, et al. HighFat Diet-Induced Obesity Rat Model: A Comparison Between Wistar and Sprague-Dawley Rat. Adipocyte (2016) 5:11-21. doi: 10.1080/21623945. 2015.1061723

34. Aranda JF, Reglero-Real N, Marcos-Ramiro B, Ruiz-Sáenz A, FernándezMartín L, Bernabé-Rubio M, et al. MYADM Controls Endothelial Barrier Function Through ERM-Dependent Regulation of ICAM-1 Expression. MBoC (2013) 24:483-94. doi: 10.1091/mbc.e11-11-0914

35. Froy O, Garaulet M. The Circadian Clock in White and Brown Adipose Tissue: Mechanistic, Endocrine, and Clinical Aspects. Endocr Rev (2018) 39:261-73. doi: 10.1210/er.2017-00193

36. Torres-Farfan C, Mendez N, Ehrenfeld P, Seron-Ferre M. In Utero Circadian Changes; Facing Light Pollution. Curr Opin Physiol (2020) 13:128-34. doi: 10.1016/j.cophys.2019.11.005 
37. Halabi D, Ehrenfeld P, Mendez N, Hans GHR, Torres-Farfan C. Fetal Programming of Adipose Tissue Function by Gestational Chronodisruption. Sleep Sci (2020) 13(Supl.2):51-8. doi: 10.5935/1984-0063.20200014

38. Tarantal A, Berglund L. Obesity and Lifespan Health-Importance of the Fetal Environment. Nutrients (2014) 6:1725-36. doi: 10.3390/nu6041725

39. Morton GJ, Schwartz MW. Leptin and the Central Nervous System Control of Glucose Metabolism. Physiol Rev (2011) 91:389-411. doi: 10.1152/ physrev.00007.2010

40. Graßmann S, Wirsching J, Eichelmann F, Aleksandrova K. Association Between Peripheral Adipokines and Inflammation Markers: A Systematic Review and Meta-Analysis: Adipokines and Inflammation: A Meta-Analysis. Obesity (2017) 25:1776-85. doi: 10.1002/oby.21945

41. Minihane AM, Vinoy S, Russell WR, Baka A, Roche HM, Tuohy KM, et al. Low-Grade Inflammation, Diet Composition and Health: Current Research Evidence and its Translation. Br J Nutr (2015) 114:999-1012. doi: 10.1017/ S0007114515002093

42. Antonelli M, Kushner I. It's Time to Redefine Inflammation. FASEB J (2017) 31:1787-91. doi: 10.1096/fj.201601326R

43. Moreno-Mendez E, Quintero-Fabian S, Fernandez-Mejia C, Lazo-de-la-VegaMonroy M-L. Early-Life Programming of Adipose Tissue. Nutr Res Rev (2020) 33(2):1-16. doi: 10.1017/S0954422420000037

44. Nehme PA, Amaral F, Lowden A, Skene DJ, Cipolla-Neto J, Moreno CRC. Reduced Melatonin Synthesis in Pregnant Night Workers: Metabolic Implications for Offspring. Med Hypotheses (2019) 132:109353. doi: 10.1016/j.mehy.2019.109353

45. Bonzini M, Palmer K, Coggon D, Carugno M, Cromi A, Ferrario M. Shift Work and Pregnancy Outcomes: A Systematic Review With Meta-Analysis of Currently Available Epidemiological Studies: Shift Work and Pregnancy Outcomes. BJOG: Int J Obstetrics Gynaecol (2011) 118:1429-37. doi: 10.1111/j.1471-0528.2011.03066.x

46. Specht IO, Hammer PEC, Flachs EM, Begtrup LM, Larsen AD, Hougaard KS, et al. Night Work During Pregnancy and Preterm Birth-A Large RegisterBased Cohort Study. PloS One (2019) 14:e0215748. doi: 10.1371/ journal.pone. 0215748

47. Martin RJ, Hausman GJ, Hausman DB. Regulation of Adipose Cell Development In Utero. Exp Biol Med (1998) 219:200-10. doi: 10.3181/ 00379727-219-44333

48. Seron-Ferre M, Reynolds H, Mendez NA, Mondaca M, Valenzuela F, Ebensperger R, et al. Impact of Maternal Melatonin Suppression on Amount and Functionality of Brown Adipose Tissue (BAT) in the Newborn Sheep. Front Endocrinol (2015) 5:232:1-11. doi: 10.3389/fendo. 2014.00232

49. Torres-Farfan C, Valenzuela FJ, Mondaca M, Valenzuela GJ, Krause B, Herrera EA, et al. Evidence of a Role for Melatonin in Fetal Sheep Physiology: Direct Actions of Melatonin on Fetal Cerebral Artery, Brown Adipose Tissue and Adrenal Gland: Functional Melatonin Receptor in Fetal
Sheep Tissues. J Physiol (2008) 586:4017-27. doi: 10.1113/jphysiol. 2008.154351

50. Touitou Y, Reinberg A, Touitou D. Association Between Light at Night, Melatonin Secretion, Sleep Deprivation, and the Internal Clock: Health Impacts and Mechanisms of Circadian Disruption. Life Sci (2017) 173:94106. doi: $10.1016 /$ j.lfs.2017.02.008

51. Carroll JE, Carrillo C, Olmstead R, Witarama T, Breen EC, Yokomizo M, et al. Sleep Deprivation and Divergent Toll-Like Receptor-4 Activation of Cellular Inflammation in Aging. Sleep (2015) 38:205-11. doi: 10.5665/sleep.4398

52. Wright KP, Drake AL, Frey DJ, Fleshner M, Desouza CA, Gronfier C, et al. Influence of Sleep Deprivation and Circadian Misalignment on Cortisol, Inflammatory Markers, and Cytokine Balance. Brain Behavior Immun (2015) 47:24-34. doi: 10.1016/j.bbi.2015.01.004

53. Lopez-Lopez J, Lopez-Jaramillo P, Camacho PA, Gomez-Arbelaez D, Cohen DD. The Link Between Fetal Programming, Inflammation, Muscular Strength, and Blood Pressure. Mediators Inflammation (2015) 2015:1-8. doi: 10.1155/2015/710613

54. Makki K, Froguel P, Wolowczuk I. Adipose Tissue in Obesity-Related Inflammation and Insulin Resistance: Cells, Cytokines, and Chemokines. ISRN Inflammation (2013) 2013:1-12. doi: 10.1155/2013/139239

55. Gatford KL, Kennaway DJ, Liu H, Kleemann DO, Kuchel TR, Varcoe TJ. Simulated Shift Work Disrupts Maternal Circadian Rhythms and Metabolism, and Increases Gestation Length in Sheep. J Physiol (2019) 597:1889-904. doi: 10.1113/JP277186

56. Edwards PD, Boonstra R. Glucocorticoids and CBG During Pregnancy in Mammals: Diversity, Pattern, and Function. Gen Comp Endocrinol (2018) 259:122-30. doi: 10.1016/j.ygcen.2017.11.012

Conflict of Interest: The authors declare that the research was conducted in the absence of any commercial or financial relationships that could be construed as a potential conflict of interest.

Publisher's Note: All claims expressed in this article are solely those of the authors and do not necessarily represent those of their affiliated organizations, or those of the publisher, the editors and the reviewers. Any product that may be evaluated in this article, or claim that may be made by its manufacturer, is not guaranteed or endorsed by the publisher.

Copyright (c) 2021 Halabi, Richter, Mendez, Kähne, Spichiger, Salazar, Torres, Vergara, Seron-Ferre and Torres-Farfan. This is an open-access article distributed under the terms of the Creative Commons Attribution License (CC BY). The use, distribution or reproduction in other forums is permitted, provided the original author(s) and the copyright owner(s) are credited and that the original publication in this journal is cited, in accordance with accepted academic practice. No use, distribution or reproduction is permitted which does not comply with these terms. 